\title{
APPROACHES TO ANALYSIS WITH INFINITESIMALS FOLLOWING ROBINSON, NELSON, AND OTHERS
}

\author{
PETER FLETCHER, KAREL HRBACEK, VLADIMIR KANOVEI, \\ MIKHAIL G. KATZ, CLAUDE LOBRY, AND SAM SANDERS
}

\begin{abstract}
This is a survey of several approaches to the framework for working with infinitesimals and infinite numbers, originally developed by Abraham Robinson in the 1960s, and their constructive engagement with the Cantor-Dedekind postulate and the Intended Interpretation hypothesis. We highlight some applications including (1) Loeb's approach to the Lebesgue measure, (2) a radically elementary approach to the vibrating string, (3) true infinitesimal differential geometry. We explore the relation of Robinson's and related frameworks to the multiverse view as developed by Hamkins.

Keywords: axiomatisations, infinitesimal, nonstandard analysis, ultraproducts, superstructure, set-theoretic foundations, multiverse, naive integers, intuitionism, soritical properties, ideal elements, protozoa.
\end{abstract}

\section{Contents}

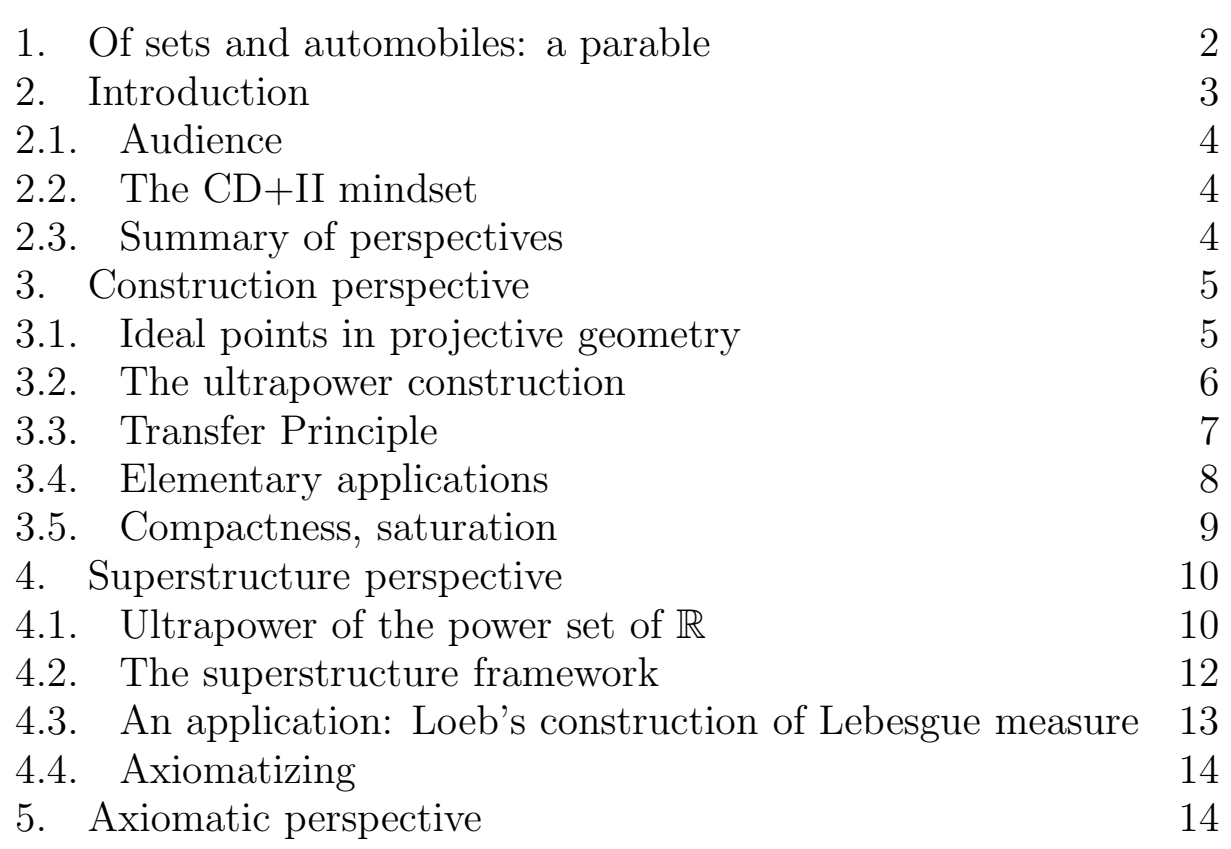


$\begin{array}{lll}5.1 . \quad \text { Nonstandard set theories } & 14\end{array}$

5.2. The axioms of BST 15

Boundedness 15

$\begin{array}{ll}\text { Bounded Idealization } & 15\end{array}$

Transfer 16

$\begin{array}{ll}\text { Standardization } & 16\end{array}$

$\begin{array}{lll}\text { 5.3. Connection with ultrafilters } & 17\end{array}$

5.4. External theories 18

5.5. Consistency, conservativity and intuitive interpretation 18

$\begin{array}{ll}\text { 5.6. The three pictures } & 19\end{array}$

5.7. The Protozoa metaphor 20

5.8. The intuitive interpretation 20

$\begin{array}{ll}\text { 5.9. Relative standardness } & 21\end{array}$

5.10. Constructive aspects of Robinson's framework 24

6. Phvsics: Radically elementary modeling 26

6.1. Tao on intricate results 26

6.2. The phvsicist's vibrating string 27

6.3. The hyperfinite vibrating string 28

6.4. Hvperfinite Brownian motion 30

7. More on Nelson 32

7.1. Kleene. Wang. Putnam. and others on the II 32

7.2. Predicate on the familiar real line 34

$\begin{array}{lll}\text { 7.3. Multiverse } & 35\end{array}$

$\begin{array}{ll}\text { 7.4. Switches } & 36\end{array}$

7.5. Historical antecedents 37

8. Reeb, naive integers, and Claim Q 39

8.1. Nonformalizable intuitions 39

8.2. Ideal intruders 40

8.3. Link between intuitionism and nonstandard analysis $\quad 40$

8.4. Nelson-stvle motivations 42

8.5. Quantum intuitions 42

9. Conclusion 43

Acknowledgments 43

References 44

\section{Of Sets And AUtomobiles: a parable}

The last third of the 19th century saw (at least) two dynamic innovations: set theory and the automobile. Consider the following parable.

A silvery family sedan is speeding down the highway. It enters heavy traffic. Every epsilon of the road requires a new delta of patience on 
the part of the passengers. The driver's inquisitive daughter Sarah 1 sitting in the front passenger seat, discovers a mysterious switch in the glove compartment. With a click, the sedan spreads wings and lifts off above the highway congestion in an infinitesimal instant. Soon it is a mere silvery speck in an infinite expanse of the sky. A short while later it lands safely on the front lawn of the family's home.

Sarah's cousin Georg'2 refuses to believe the story: true Sarah's father is an NSA man, but everybody knows that Karl Benz's 1886 PatentMotorwagen had no wing option!

At a less parabolic level, some mathematicians feel that, on the one hand, "It is quite easy to make mistakes with infinitesimals, etc." (Quinn [137, p. 31]) while, as if by contrast, "Modern definitions are completely selfcontained, and the only properties that can be ascribed to an object are those that can be rigorously deduced from the definition... Definitions that are modern in this sense were developed in the late 1800s." (ibid., p. 32).

We will have opportunity to return to Sarah, cousin Georg, switches, and the heroic "late 1800s" in the sequel; see in particular Section 7.4.

\section{INTRODUCTION}

The framework created by Abraham Robinson in the 1960s and called by him "nonstandard analysis" is an active research area featuring many applications in other fields 3 its own journal (Journal of Logic and Analysis) 4 and high-profile advocates like Terry Tao:5 see e.g., Tao [160], Tao-Vu [161]. The time may be ripe for a survey of some of the approaches to the field.

\footnotetext{
${ }^{1}$ For the literary career of this character see Kanovei-Katz-Schaps 86 .

${ }^{2}$ This character similarly made a (cameo) appearance in the final section of Kanovei-Katz-Schaps [86, pp. 17-18].

${ }^{3}$ See Section 6] and Stroyan-Luxemburg [157, Henle-Kleinberg [68, Arkeryd [4, Lutz-Goze 120, Keisler [106, Albeverio et al. 11, Wallet [168, van den Berg [163, Callot 29, Rubio [146, Diener-Diener [36, Henson [69, Ross [145, Jin [83, Kanovei-Shelah [93, Kanovei-Lyubetskii [89], Goldbring [52], van den DriesGoldbring [165, Nowik-Katz [129], Pražák-Slavík [135]; this list of applications is by no means comprehensive.

${ }^{4}$ See http://www.logicandanalysis.org

${ }^{5}$ The field has also had its share of high-profile detractors like Errett Bishop 22 and Alain Connes 32. Their critiques were analyzed in Katz-Katz 95, Katz-Leichtnam [98, Kanovei-Katz-Mormann [88, and Sanders [150]; see also material in Section [5.10 around note 30. For further details on Connes and the taming of differential geometry see note 38. Additional criticisms were voiced by J. Earman [40, K. Easwaran [41, H. M. Edwards [42, G. Ferraro [45, J. Grabiner [56], J. Gray [57, P. Halmos 60], H. Ishiguro [82], K. Schubring [151,
} 
2.1. Audience. The text presupposes some curiosity about infinitesimals in general and Robinson's framework in particular. While the text is addressed to a somewhat informed audience not limited to specialists in the field, an elementary introduction is provided in Section 3 .

Professional mathematicians and logicians curious about Robinson's framework, as well as mathematically informed philosophers are one possible (proper) subset of the intended audience, as are physicists, engineers, and economists who seem to have few inhibitions about using terms like infinitesimal and infinite number 6

2.2. The CD+II mindset. A survey of this sort necessarily enters into a relationship of constructive (if not subversive) engagement with a pair of assumptions commonly held among mathematicians, namely

(1) the Cantor-Dedekind postulate (CD) identifying the line in physical space with the real number line (see Ehrlich [43] and Katz-Katz [96]), and

(2) the Intended Interpretation hypothesis (II), entailing an identification of a standard $\mathbb{N}$ in its set-theoretic context, on the one hand, with ordinary intuitive counting numbers, on the other.

We will deal with the II in more detail in Section 7.1. How the engagement with the CD+II mindset plays itself out should become clearer in the sequel.

2.3. Summary of perspectives. This article was inspired in part by the posting of Joel Hamkins at Math Overflow [65]. Hamkins wrote: "There are at least three distinct perspectives one can naturally take on when undertaking work in nonstandard analysis. In addition, each of these perspectives can be varied in two other dimensions, independently. Those dimensions are, first, the order of nonstandardness (whether one wants nonstandardness only for objects, or also for functions and predicates, or also for sets of functions and sets of those and so on); and second, how many levels of standardness and nonstandardness one desires."

We shall describe the three perspectives and discuss their similarities and differences in Sections 3 through 5 . The breakdown into three main perspectives parallels that in Bair-Henry [9]. Sections 6 through 8

Y. Sergeyev [152], and D. Spalt [156]. These were dealt with respectively in KatzSherry [102, Bascelli et al. [15], Kanovei-Katz-Sherry [87, Bair et al. [7, BorovikKatz [28, Błaszczyk et al. [25], Błaszczyk et al. [23], Bascelli et al. [16], Błaszczyk et al. [26], Gutman et al. [58, Katz-Katz [94].

${ }^{6}$ See e.g., a quotation in Delfini-Lobry 35] from Berkeley Physics Course, Crawford 33 and Section 6.2. 
explore additional aspects of Robinson's framework. The survey BenciForti-Di Nasso [19] was an earlier effort in this general direction.

\section{Construction perspective}

Hamkins wrote.7 "In this perspective, one thinks of the nonstandard universe as the result of an explicit construction. In the most basic instance, one starts with the familiar ordered field of real numbers $\langle\mathbb{R},+, \times,<, 0,1\rangle$ and extends it to a larger ordered field."

The larger field then necessarily contains infinitesimals and infinitely large numbers. Constructing such an extension by itself is easy, 8 but not just any extension will do. In order for it to serve as a tool for analysis, one needs at least to be able to extend every function $f$ of interest defined on $\mathbb{R}$ to a function ${ }^{*} f$ defined on the larger field so that ${ }^{*} f \bigsqcup_{\mathbb{R}}=f$. This extension is expected to preserve the important properties of $f$ (see Section 3.3 for an introductory discussion of the Transfer Principle). We will sketch an ultrapower construction of such a hyperreal extension $\mathbb{R} \hookrightarrow{ }^{*} \mathbb{R}$.

3.1. Ideal points in projective geometry. As a motivational comment, it may be helpful to compare extending the field $\mathbb{R}$ to adding ideal points at infinity in projective geometry 9 In projective geometry, an affine plane defined by the equation $a x+b y+c z=d$ is outfitted with an assortment of ideal points at infinity, one for each pencil of parallel lines in the plane. The new points are viewed as satisfying the "same" equation once one introduces homogeneous coordinates $a x_{1}+b x_{2}+c x_{3}=d x_{4}$. In addition to ideal points being added to the ambient space, each substructure, namely each line, is similarly enriched by the addition of one of these points. This is analogous to a hyperreal extension presented below where not only the extension ${ }^{*} \mathbb{R}$ itself has additional points, but every object, such as subset, or function, is similarly enriched.

At the beginning of the 17th century Johannes Kepler invoked a principle of continuity to justify (at least) two distinct procedures: a

\footnotetext{
${ }^{7}$ The quotations in this section have been slightly edited.

${ }^{8}$ The simplest example of such an extension is the field of rational functions (quotients of polynomials with real coefficients), linearly ordered by defining $f<g$ if and only if $f(x)<g(x)$ for all sufficiently large $x$. Then real numbers are embedded in it as constant functions, the function $f(x)=\frac{1}{x}$ represents an infinitesimal, and the function $g(x)=x$ represents an infinitely large number. More advanced examples are provided by Levi-Civita fields; see Lightstone-Robinson [112.

${ }^{9} \mathrm{Such}$ an analogy is meaningful in the context of ordered fields, whereas the addition of points at infinity in projective geometry makes sense for an arbitrary field.
} 
continuous sweep of all conics aided by ideal points at infinity (a prototype of the modern theorem that all conics are projectively equivalent), and a view of a circle (or more general curves) as an infinite-sided polygon. The latter view found an avid adherent in the person of Gottfried Leibniz, whose law of continuity postulated that the rules valid in the finite realm remain valid in the infinite realm; see Section 7.5 and Katz-Sherry [102] for additional details.

3.2. The ultrapower construction. Let $\mathbb{R}^{\mathbb{N}}$ denote the ring of infinite sequences of real numbers, with arithmetic operations defined termwise. Then we have a totally ordered field

$$
* \mathbb{R}=\mathbb{R}^{\mathbb{N}} / \mathrm{MAX}
$$

where "MAX" is a suitable maximal ideal. To produce such a maximal ideal, one can exploit a finitely additive measure $\xi$,

$$
\xi: \mathcal{P}(\mathbb{N}) \rightarrow\{0,1\}
$$

(thus $\xi$ takes only two values, 0 and 1 ) taking the value 1 on each cofinite set, where $\mathcal{P}(\mathbb{N})$ is the set of subsets of $\mathbb{N}$. For each pair of complementary subsets of $\mathbb{N}$, such a measure $\xi$ "decides" in a coherent way which one is "negligible" (i.e., of measure 0) and which is "dominant" (measure 1).

The ideal MAX consists of all "negligible" sequences $\left\langle u_{n}\right\rangle$, i.e., sequences which vanish for a set of indices of measure 1, namely,

$$
\xi\left(\left\{n \in \mathbb{N}: u_{n}=0\right\}\right)=1 .
$$

The subset $U_{\xi} \subseteq \mathcal{P}(\mathbb{N})$ whose members are sets of measure 1 is called a free (or nonprincipal) ultrafilter.

Note the formal analogy between (3.1) and the construction of the real numbers as equivalence classes of Cauchy sequences of rational numbers. In both cases, the subfield is embedded in the superfield by means of the constant sequences, and the ring of sequences is factored by a maximal ideal.

Elements of $* \mathbb{R}$ are called hyperreal numbers. The field $\mathbb{R}$ is embedded into ${ }^{*} \mathbb{R}$ via a mapping that assigns to each $r \in \mathbb{R}$ the hyperreal ${ }^{*} r$, namely, the equivalence class of the constant sequence with value $r$; we shall identify $r$ and ${ }^{*} r$. The equivalence classes of sequences with terms from $\mathbb{N}$ form the set ${ }^{*} \mathbb{N}$ of hypernatural numbers. The equivalence class of the sequence $\langle n: n \in \mathbb{N}\rangle$ (respectively, $\left\langle\frac{1}{n}: n \in \mathbb{N}\right\rangle$ ) is an infinitely large integer (respectively, an infinitesimal).

The order ${ }^{*}<$ on ${ }^{*} \mathbb{R}$ is defined by setting

$$
\left[\left\langle u_{n}\right\rangle\right]^{*}<\left[\left\langle v_{n}\right\rangle\right] \text { if and only if } \xi\left(\left\{n \in \mathbb{N}: u_{n}<v_{n}\right\}\right)=1 .
$$


This order extends the ordering $<$ of $\mathbb{R}$ as follows: for $r, s \in \mathbb{R}$, we have $r<s$ if and only if $r^{*}<s$ (that is, ${ }^{*} r^{*}<{ }^{*} s$ ). Hence we can drop the asterisk on $<$ with no risk of confusion.

Addition ${ }^{*}+$ on ${ }^{*} \mathbb{R}$ is defined by

$$
\left[\left\langle u_{n}\right\rangle\right]^{*}+\left[\left\langle v_{n}\right\rangle\right]=\left[\left\langle u_{n}+v_{n}\right\rangle\right]
$$

and similarly for multiplication. These operations extend the corresponding operations on $\mathbb{R}$, and hence the asterisks can and shall be dropped. Moreover, ${ }^{*} \mathbb{R}$, with these operations and ordering, is an ordered field extending $\mathbb{R}$.

The equivalence classes of sequences with rational terms form the subfield $* \mathbb{Q}$ consisting of hyperrational numbers.

A hyperreal number $x$ is finite (or limited) if $-r<x<r$ for some $r \in \mathbb{R}$, and infinitesimal if $-r<x<r$ for all $r \in \mathbb{R}, r>0$. Every finite hyperreal number $x$ rounds off to the nearest real number, called its shadow (or standard part) and denoted $\mathbf{s h}(x)$; here the difference $x-$ $\operatorname{sh}(x)$ is infinitesimal.

Similarly, the ultrapower construction allows one to extend arbitrary functions and relations from $\mathbb{R}$ to ${ }^{*} \mathbb{R}$. Every function $f: \mathbb{R} \rightarrow \mathbb{R}$ has a natural extension ${ }^{*} f:{ }^{*} \mathbb{R} \rightarrow{ }^{*} \mathbb{R}$ acting componentwise: ${ }^{*} f\left(\left[\left\langle u_{n}\right\rangle\right]\right)=$ $\left[\left\langle f\left(u_{n}\right)\right\rangle\right]$. If $S \subseteq \mathbb{R}$, then the natural extension of $S$, denoted ${ }^{*} S$, is defined by

$$
\left[\left\langle u_{n}\right\rangle\right] \in{ }^{*} S \text { if and only if } \xi\left(\left\{n \in \mathbb{N}: u_{n} \in S\right\}\right)=1
$$

similarly for relations on $\mathbb{R}$. The asterisks are habitually dropped when there is no risk of confusion; typically they are dropped for functions and operations, but not for sets.

One of the first treatments of an ultrapower-type construction appeared in Hewitt [71].

3.3. Transfer Principle. The Transfer Principle is a type of theorem that, depending on the context, asserts that rules, laws or procedures valid for a certain number system, still apply (i.e., are "transfered") to an extended number system. Thus, the familiar extension $\mathbb{Q} \hookrightarrow \mathbb{R}$ preserves the property of being an ordered field. To give a negative example, the extension $\mathbb{R} \hookrightarrow \mathbb{R} \cup\{ \pm \infty\}$ of the real numbers to the so-called extended reals does not preserve the field properties. the extension $\mathbb{R} \hookrightarrow \mathbb{C}$ preserves the field axioms, but does not preserve the property of not having a square root of -1 .

The hyperreal extension $\mathbb{R} \hookrightarrow{ }^{*} \mathbb{R}$ preserves all first-order properties. The result in essence goes back to Łoś [119].

For example, the identity $\sin ^{2} x+\cos ^{2} x=1$ remains valid for all hyperreal $x$, including infinitesimal and infinite inputs $x \in{ }^{*} \mathbb{R}$. Another 
example of a transferable property is the property that

$$
\text { for all positive } x, y \text {, if } x<y \text { then } \frac{1}{y}<\frac{1}{x} \text {. }
$$

The Transfer Principle applies to formulas like that characterizing the continuity of a function $f: \mathbb{R} \rightarrow \mathbb{R}$ at a point $c \in \mathbb{R}$ :

$$
(\forall \varepsilon>0)(\exists \delta>0)(\forall x)[|x-c|<\delta \Rightarrow|f(x)-f(c)|<\varepsilon] ;
$$

namely, formulas that quantify over elements of $\mathbb{R}$. See Lindstrøm [113], Goldblatt [51, and Gordon-Kusraev-Kutateladze [55] for additional details.

3.4. Elementary applications. Let $H$ be an infinite hypernatural number (more formally, $H \in{ }^{*} \mathbb{N} \backslash \mathbb{N}$ ) and $z \in \mathbb{C}$. We retrieve formulas of the sort that already appeared in Euler; see Bair et al. [7]. In the following we will exploit hypercomplex numbers ${ }^{*} \mathbb{C}={ }^{*} \mathbb{R}+\sqrt{-1} * \mathbb{R}$. For example we obtain

$$
e^{z} \approx\left(1+\frac{z}{H}\right)^{H}
$$

where $\approx$ is the relation of infinite proximity 10 In particular, the identity $e^{i \pi}=-1$ takes the form

$$
\left(1+\frac{i \pi}{H}\right)^{H} \approx-1
$$

Since the principal branch of $z \mapsto H \sqrt[H]{z}$ is Lipschitz near $z=-1$, it preserves the relation of infinite proximity there 11 Therefore the relation (3.2) implies

$$
H \sqrt[H]{-1} \approx H+i \pi
$$

yielding the following formula for $\pi$ :

$$
\pi \approx \frac{H \sqrt[H]{-1}-H}{\sqrt{-1}}
$$

This expression for $\pi$ can be interpreted as the derivative of $(-1)^{x}$ at $x=-\frac{1}{2} \cdot 12$

\footnotetext{
${ }^{10}$ Here $z \approx w$ if and only if $|z-w|$ is infinitesimal.

${ }^{11}$ Here we use the principal branch of the root. The point is that the Lipschitz constant for the internal function in question can be chosen finite, since the Lipschitz constant does not depend on the index assuming that we work in a small neighborhood of -1 . Therefore the Lipschitz property with the same constant holds by the Transfer Principle (see Section 3.3) for all infinite $H$, as well.

Alternatively, we have the primitive root $\sqrt[H]{-1}=\cos \frac{\pi}{H}+i \sin \frac{\pi}{H}$, hence $H \sqrt[H]{-1}=$ $H \cos \frac{\pi}{H}+i H \sin \frac{\pi}{H} \simeq H+i \pi$ since $\cos \frac{\pi}{H} \simeq 1$ and $\frac{H}{\pi} \sin \frac{\pi}{H} \simeq 1$.

${ }^{12}$ The derivative is computed from the definition, using the infinitesimal $\frac{1}{H}$. The derivative of $(-1)^{x}$ is $(\ln (-1))(-1)^{x}$. We substitute $x=-\frac{1}{2}$ to obtain $\pi$ since $\ln (-1)=\pi i$ and $(-1)^{-1 / 2}=-i$.
} 
To mention another elementary application, the heuristic principle that the period of small oscillations of the pendulum is independent of their amplitude finds precise mathematical implementation for oscillations with infinitesimal amplitude Kanovei-Katz-Nowik [85]. A transparent treatment of the Jordan curve theorem exploiting Robinson's framework appears in Kanovei-Reeken [91]. For additional applications see Section [6]

3.5. Compactness, saturation. We have described the ultrapower construction with respect to a fixed free ultrafilter $U$ on the natural numbers. Another way to implement these ideas is to use the compactness theorem of first-order logic, as Robinson originally did in [141].

In some applications of nonstandard analysis one may wish to employ stronger saturation properties (see below) than those satisfied by (3.1); this can usually be accomplished by using special ultrafilters on larger index sets.

The property of saturation of a hyperreal field is analogous to compactness in classical analysis, and can be expressed as follows.

Definition 3.1. A hyperreal field ${ }^{*} \mathbb{R}$ is countably saturated if every nested infinite sequence of nonempty internal sets (see Sections 4.1 and 4.2) has a common element.

More generally, a structure is called $\kappa$-saturated if for every collection of properties (expressible in the language of the structure) of cardinality strictly less than $\kappa$, each finite subcollection of which is satisfied by some element of the structure, there is an element of the structure that satisfies all of the properties simultaneously. Note that (3.1) is always countably saturated 14

The existence of a common point for a decreasing nested sequence of compact sets $\left\langle K_{n}: n \in \mathbb{N}\right\rangle$ can be seen as a special case of the saturation property. Indeed, the decreasing nested sequence of internal sets, $\left\langle{ }^{*} K_{n}: n \in \mathbb{N}\right\rangle$, has a common point $x$ by saturation. But for a compact set $K_{n}$, every point of ${ }^{*} K_{n}$ is nearstandard (i.e., infinitely close to a point of $\left.K_{n}\right)$. In particular, $\operatorname{sh}(x) \in K_{n}$ for all $n$, as required.

Hamkins wrote in [65]: "To give a sample consequence of saturation, we observe that every infinite graph, no matter how large, arises as an induced subgraph of a hyperfinite graph in any sufficiently saturated model of nonstandard analysis. This often allows one to undertake

\footnotetext{
${ }^{13}$ See also the references provided in note 3 ,

${ }^{14}$ Traditionally, countable saturation is the same as $\aleph_{1}$-saturation, because a collection of cardinality strictly less than $\aleph_{1}$ is the same as a countable (including finite, which is a trivial case anyway) collection.
} 
finitary constructions with infinite graphs, modulo the move to a nonstandard context."

"The ultrapower construction can be extended to the power set of $\mathbb{R}$ and its higher iterates 15 In the end, one realizes that one might as well take the ultrapower of the entire set-theoretic universe $V$. One then has a copy of the standard universe $V$ inside the nonstandard realm ${ }^{*} V$, which one analyzes and understands by means of the ultrapower construction itself 16 A few applications of nonstandard analysis exploit not just a single ultrapower, but an ultrapower construction repeated along some linear order. Such iterated ultrapower constructions give rise to many levels of nonstandardness, a useful feature. Ultimately one is led to adopt all of model theory as one's toolkit." [65]

\section{Superstructure Perspective}

As Hamkins points out, before long, one wishes nonstandard analogues of the power set $\mathcal{P}(\mathbb{R})$ and its higher iterates. We will now implement this idea.

4.1. Ultrapower of the power set of $\mathbb{R}$. Elements of the ultrapower of $\mathcal{P}(\mathbb{R})$ are the equivalence classes of sequences $\left\langle A_{n}: n \in \mathbb{N}\right\rangle$ of subsets $A_{n} \subseteq \mathbb{R}$ where sequences $\left\langle A_{n}\right\rangle$ and $\left\langle B_{n}\right\rangle$ are defined to be equivalent if and only if we have $\left\{n \in \mathbb{N}: A_{n}=B_{n}\right\} \in U_{\xi}$.

The relation ${ }^{*} \in$ between $x=\left[\left\langle x_{n}\right\rangle\right]$ in ${ }^{*} \mathbb{R}$ and $\left[\left\langle A_{n}\right\rangle\right]$ in the ultrapower of $\mathcal{P}(\mathbb{R})$ is defined by setting

$$
x^{*} \in\left[\left\langle A_{n}\right\rangle\right] \text { if and only if }\left\{n \in \mathbb{N}: x_{n} \in A_{n}\right\} \in U_{\xi} .
$$

In this construction, the sets in the ultrapower of $\mathcal{P}(\mathbb{R})$ are not subsets of ${ }^{*} \mathbb{R}$ (they are equivalence classes of sequences), and the membership relation ${ }^{*} \in$ is not the usual membership relation $\in$. Both of these problems are solved by the following stratagem. With each equivalence class $\left[\left\langle A_{n}: n \in \mathbb{N}\right\rangle\right]$ in the ultrapower of $\mathcal{P}(\mathbb{R})$ we associate a subset $A$ of $* \mathbb{R}$ as follows:

$$
x \in A \text { if and only if } \quad x^{*} \in\left[\left\langle A_{n}\right\rangle\right] .
$$

The subsets of ${ }^{*} \mathbb{R}$ associated with the members of the ultrapower of $\mathcal{P}(\mathbb{R})$ in this way are called internal sets. The collection of all internal subsets of ${ }^{*} \mathbb{R}$ is denoted ${ }^{*} \mathcal{P}(\mathbb{R})$. We have ${ }^{*} \mathcal{P}(\mathbb{R}) \varsubsetneqq \mathcal{P}\left({ }^{*} \mathbb{R}\right)$. The

\footnotetext{
${ }^{15}$ See Section 4

${ }^{16}$ However, this idea runs into technical issues. The standard universe $V$ is not a set, and the ultrapower of the membership relation in $V$ is not well-founded. We discuss the ways in which nonstandard analysis deals with these issues in Section 5 .
} 
inclusion is strict because there are subsets of $* \mathbb{R}$ that are not internal; for example, the subset $\mathbb{R} \subseteq{ }^{*} \mathbb{R}$ is not internal.

In the special case when $A_{n}=S \subseteq \mathbb{R}$ for all $n \in \mathbb{N}$, the associated internal set is the natural extension of $S$ introduced earlier and denoted ${ }^{*} S$. We shall also refer to ${ }^{*} S$ as the standard copy of $S$.

Definition 4.1. A hyperfinite set $X$ is an internal set associated with the equivalence class of a sequence $\left\langle A_{n}: n \in \mathbb{N}\right\rangle$ where each $A_{n}$ is finite.

Such a set admits an internal enumeration by a hypernatural number, denoted $|X|$. In more detail, the hypernatural number $|X|$ is the coset of the sequence of integers $\left|A_{n}\right|$ where $\left|A_{n}\right|$ is the ordinary number of elements in the finite set $A_{n}$.

It can be shown that every subset of ${ }^{*} \mathbb{R}$ definable in ${ }^{*} \mathbb{R} \cup * \mathcal{P}(\mathbb{R})$ from internal parameters is internal, a fact known as the internal definition principle. Thus, if one defines a new object by a formula exploiting only internal objects, the new object is necessarily internal, as well. For instance, if one defines $T$ to be the set of integers between 1 and an infinite hyperinteger $H$, then $T$ is necessarily internal. Thus one needn't specify a presentation of the internal set $T$ with respect to the ultrapower construction.

The Transfer Principle now applies to formulas that quantify over both elements and sets of elements of $\mathbb{R}$. Such statements transfer into statements true about all internal sets, but not necessarily about all sets. Thus for example every nonempty internal set of hyperreals bounded above has a supremum in ${ }^{*} \mathbb{R}$, but not every set of hyperreals does 17

This construction can easily be extended to the second and higher iterates of the power set operation. But the few examples already given indicate that, for the practice of analysis, it suffices to know that an extension of the standard structure exists, with suitable properties, such as the Transfer Principle; the actual construction is of secondary importance.

The superstructure framework enables one to step back from the details of the actual (ultrapower) construction 18 It is still the most popular vehicle for the practice of nonstandard analysis; see for example

\footnotetext{
${ }^{17}$ The subset $\mathbb{R} \subseteq{ }^{*} \mathbb{R}$ is a counterexample: it is bounded above by every positive infinitely large number $L$, but it does not have a least upper bound: if $L$ is an upper bound for $\mathbb{R}$, then $L-1$ is similarly an upper bound.

${ }^{18}$ Such a perspective is comparable to the way mathematicians think of real numbers. They certainly wish to know that real numbers can be conceived as equivalence classes of Cauchy sequences of rationals. However, he also wishes to be able to ignore the actual construction of $\mathbb{R}$ entirely in his everyday work, and to
} 
Robinson-Zakon [144], Chang-Keisler [30], Albeverio et al. [1]. We describe it in the next subsection.

4.2. The superstructure framework. For any set of individuals $X$, the superstructure over $X$ is obtained from $X$ by taking the power set countably many times. In more detail, one defines the superstructure recursively by setting $V_{0}(X)=X, V_{n+1}(X)=V_{n}(X) \cup \mathcal{P}\left(V_{n}(X)\right)$ for $n \in \omega$, and $V_{\omega}(X)=\bigcup_{n<\omega} V_{n}(X)$.

The superstructure framework for nonstandard analysis consists of two superstructures, $V_{\omega}(X)$ and $V_{\omega}(Y)$ where $X \varsubsetneqq Y$ are infinite sets, and a mapping $*: V_{\omega}(X) \rightarrow V_{\omega}(Y)$ such that ${ }^{*} a=a$ for all $a \in$ $X,{ }^{*} X=Y$, and $A \in B$ implies ${ }^{*} A \in{ }^{*} B$ for $A, B \in V_{\omega}(X)$. From now on, we take $X=\mathbb{R}$. This facilitates comparison with the construction perspective; meanwhile the discussion in this section applies fully to the general situation. Note that $V_{\omega}(\mathbb{R})$ contains all objects of interest to classical analysis, such as the fields of real and complex numbers, the higher-dimensional spaces $\mathbb{R}^{n}$ and $\mathbb{C}^{n}$, functions on these, collections of functions, Hilbert spaces $\ell^{2}$ and $L^{2}$, functionals, etc.

We shall refer to $V_{\omega}(\mathbb{R})$ as the standard universe and to $V_{\omega}\left({ }^{*} \mathbb{R}\right)$ as the nonstandard universe. Thus, every set $S$ in the standard universe $V_{\omega}(\mathbb{R})$ has a standard copy ${ }^{*} S$ in the nonstandard universe (cf. Sections 3.2 and 4.1). The sets contained in $V_{\omega}\left({ }^{*} \mathbb{R}\right)$ that are elements of some ${ }^{*} S$ for $S \in V_{\omega}(\mathbb{R})$ are called internal, and ${ }^{*} V_{\omega}(\mathbb{R})$ denotes the collection containing the elements of ${ }^{*} \mathbb{R}$ together with all internal sets; we refer to it as the internal universe. Note that ${ }^{*} V_{\omega}(\mathbb{R})=\bigcup_{n<\omega}{ }^{*} V_{n}(\mathbb{R})$. We have a proper inclusion ${ }^{*} V_{\omega}(\mathbb{R}) \subsetneq V_{\omega}\left({ }^{*}\right)$; for example, $\mathbb{R} \subseteq{ }^{*} \mathbb{R}$ is not an internal set. Obviously, $V_{\omega}(\mathbb{R}) \subseteq V_{\omega}\left({ }^{*} \mathbb{R}\right)$, but all sets in $V_{\omega}(\mathbb{R})$, except for the hereditarily finite ones, are external (not internal).

The main principle that connects the two superstructures is the Transfer Principle, which posits that any property expressible in the language of the superstructures by a bounded quantifier formula 19 holds in the standard universe $V_{\omega}(\mathbb{R})$ about some objects if and only if it holds for the standard copies of those objects in the internal universe ${ }^{*} V_{\omega}(\mathbb{R})$ or, equivalently, in the nonstandard universe $V_{\omega}\left({ }^{*} \mathbb{R}\right)$.

By the Transfer Principle, $\left\langle{ }^{*} \mathbb{R},{ }^{*}+,{ }^{*} \times,{ }^{*}<, 0,1\right\rangle$ is a field of hyperreals with the properties as in Section 3.2 . In particular, ${ }^{*} \mathbb{R}$ contains nonstandard numbers, such as infinitesimals and infinitely large numbers.

consider real numbers as individuals (atomic entities) and $\mathbb{R}$ simply as a complete ordered field.

${ }^{19} \mathrm{~A}$ bounded quantifier formula is a formula where all quantifiers have the form $(\exists x \in y)$ or $(\forall x \in y)$. 
Many arguments in nonstandard analysis rely on a saturation principle (see Definition 3.1). Two useful forms of saturation available in the superstructure framework are the following:

(1) If $A_{1} \supseteq A_{2} \supseteq \ldots$ are nonempty internal sets, then $\bigcap_{n<\omega} A_{n} \neq \varnothing$.

(2) If $\left\{A_{i}: i \in I\right\}$ is a collection of sets in $V_{\omega}(\mathbb{R})$ possessing the finite intersection property, then $\bigcap_{i \in I}{ }^{*} A_{i} \neq \varnothing$.

We shall not give any details of the construction of superstructure frameworks. The advantage of this approach lies precisely in the fact that there is no need to do so. One can accept the framework and proceed to use it in any area of mathematics, while leaving its construction to specialists. Such constructions proceed along the lines of Section 4.1, see [30]. The internal universe ${ }^{*} V_{\omega}(\mathbb{R})$ is isomorphic to the union of the ultrapowers of $V_{n}(\mathbb{R})$ for all $n \in \omega, 20$ the nonstandard universe $V_{\omega}\left({ }^{*} \mathbb{R}\right)$ contains additional, external sets.

\subsection{An application: Loeb's construction of Lebesgue measure.}

As an example of the interplay between the various kinds of sets in the superstructure, we describe a nonstandard construction of the Lebesgue measure on $[0,1]$ due to P. Loeb [117] (with an improvement that seems to have appeared first in Hrbacek [75]).

We fix an infinite integer $N \in{ }^{*} \mathbb{N}$. Let $t_{i}=\frac{i}{N}$ for $i=0, \ldots, N$. The hyperfinite set $T=\left\{t_{0}, t_{1}, \ldots, t_{N}\right\}$ (see the sentence following Definition 4.1) is referred to as "hyperfinite time line" in Albeverio et al. [1]. Let $\mu$ be the counting measure on $T$, i.e., $\mu(X)=|X| /|T|$ for each internal set $X \subseteq T$; then $\mu$ itself is also internal. For every $A \subseteq$ $[0,1]$, the set

$$
\mathbf{s h}^{-1}[A]=\left\{z \in *^{*}[0,1]: \mathbf{s h}(z) \in A\right\}
$$

is the, generally external, set of all $z \in{ }^{*}[0,1]$ such that $z \approx x$ for some $x \in A$. Finally, we define

$$
m_{L}(A)=\inf \left\{\operatorname{sh}(\mu(X)): X \subseteq T \text { is internal and } \operatorname{sh}^{-1}[A] \cap T \subseteq X\right\} .
$$

The collection in braces is a (nonempty, bounded below) subset of $\mathbb{R}$, so the infimum exists. It can be shown (see [1] for some details) that $m_{L}$ is the Lebesgue outer measure on $[0,1]$; in particular, a set $A \subseteq[0,1]$ is Lebesgue measurable if and only if one has $m_{L}(A)+m_{L}([0,1] \backslash A)=1$, and in this case $m_{L}(A)$ is precisely the Lebesgue measure of $A$.

\footnotetext{
${ }^{20}$ More precisely, to the bounded ultrapower of $V_{\omega}(\mathbb{R})$.
} 
4.4. Axiomatizing. It is often thought worthwhile (for example, for pedagogical reasons; see [99]) to develop the subject purely from general principles that make the nonstandard arguments succeed. This approach is similar to, say, deriving results from the axioms for algebraically closed fields rather than arguing about these mathematical structures directly. As an example of such an exposition, Keisler's calculus textbook is based on five simple axioms A through E that hold in every superstructure framework; see Keisler [107] and [105].

The next step would be to take the entire universe of sets, $V$, as a "superstructure." We already indicated that this move gives rise to some issues, both technical and fundamental 21 It is considered in the next section.

\section{Axiomatic Perspective}

5.1. Nonstandard set theories. As mentioned above, taking an ultrapower of the entire set-theoretic universe $V$ is an attractive idea. Since $V$ is not a set in ZFC, the best way to handle this construction is axiomatic. The earliest axiomatizations of ultrapowers of $V$ were proposed by Petr Vopěnka in [167] as part of his project of axiomatizing the method of forcing. The first nonstandard set theories that extend ZFC and can serve as a framework for the practice of nonstandard analysis were developed independently by Hrbacek [74] and Nelson [127]22 We refer to Kanovei-Reeken 92 for a comprehensive survey of the field.

Nonstandard set theories typically possess the set membership predicate $\epsilon$ and a unary predicate of standardness st; here $\mathbf{s t}(x)$ reads " $x$ is standard." The analog of $\mathbf{s t}(x)$ in the superstructure framework is " $x$ is a standard copy."

A helpful classification tool is a distinction between internal and external set theories.

Internal set theories axiomatize only the standard and internal sets. Edward Nelson's IST is the best known example. The theory IST postulates all the axioms of ZFC in the pure $\in$-language, together

\footnotetext{
${ }^{21}$ While the ultrapower of $V_{n}(\mathbb{R})$ for $n \in \omega$ is well-founded, and hence isomorphic to a transitive set, by the Mostowski collapse lemma, when one takes the ultrapower of $V_{\omega}(\mathbb{R})$, or even of the entire $V$, the membership relation ${ }^{*} \in$ is non-well-founded, and therefore the ultrapower is not isomorphic to a transitive set or class in the framework of ZFC. This situation calls for an ontological commitment beyond what is required by $\mathrm{ZFC}$, and is best handled axiomatically.

${ }^{22}$ Vopernka also developed a nonstandard set theory that enables infinitesimal methods. His Alternative Set Theory (AST) is incompatible with ZFC and is not considered here. See Sochor [155] and Vopěnka [166.
} 
with three extra axioms (more precisely, axiom schemata) of Idealization, Standardization, and Transfer, which govern the relations between standard and nonstandard sets. The theory is particularly attractive because of its simplicity. Kanovei [84] modified IST by limiting its universe to bounded sets 23 The resulting internal theory $\mathrm{BST}^{24}$ provides the same mathematical tools as IST, but it has better metamathematical behavior; see Section 5.5.

5.2. The axioms of BST. We shall now formulate the axioms of BST. The notation $\left(\exists^{\mathbf{s t}} x\right) \ldots$ and $\left(\forall^{\mathbf{s t}} x\right) \ldots$ abbreviates respectively the formulas $(\exists x)(\operatorname{st}(x) \wedge \ldots)$ and $(\forall x)(\operatorname{st}(x) \Rightarrow \ldots)$. The notation $\left(\forall^{\operatorname{stfin}} a\right) \ldots$ is shorthand for the formula

$$
(\forall a)((\operatorname{st}(a) \wedge(a \text { is finite })) \Rightarrow \ldots) \text {. }
$$

The axioms of BST include those of ZFC (with Separation and Replacement only for formulas in the $\in$-language) together with the following four additional principles.

\section{Boundedness.}

$(\forall x)\left(\exists^{\mathrm{st}} y\right)(x \in y)$.

This means that every set is an element of some standard set. This is the main difference between BST and IST. In IST there is a set that contains all standard sets as elements.

\section{Bounded Idealization.}

$\left(\forall^{\text {st }} A\right)\left[\left(\forall^{\text {stfin }} a \subseteq A\right)(\exists y)(\forall x \in a) \Phi(x, y) \equiv(\exists y)\left(\forall^{\text {st }} x \in A\right) \Phi(x, y)\right]$ where $\Phi(x, y)$ is any formula in the $\in$-language, possibly with parameters.

This axiom is a version of saturation or compactness; see Definition 3.1. Loosely speaking, if a collection of properties $\Phi(x, y), x \in A$ is finitely satisfiable, that is, for every standard finite set $a=\left\{x_{1}, \ldots, x_{n}\right\}$ of elements of $A$ there is some $y$ such that $\Phi\left(x_{1}, y\right) \wedge \ldots \wedge \Phi\left(x_{n}, y\right)$ holds, then there is some $y$ that satisfies $\Phi(x, y)$ simultaneously for all standard $x \in A$. As a basic example, consider the property

$$
(i, j \in \mathbb{N}) \wedge(i<j) \text {. }
$$

For every standard finite set $a=\left\{i_{1}, \ldots, i_{n}\right\} \subseteq \mathbb{N}$ there is $j \in \mathbb{N}$ such that $\left(i_{1}<j\right) \wedge \ldots \wedge\left(i_{n}<j\right)$ (namely, take $\left.j=\max \left\{i_{1}, \ldots, i_{n}\right\}+1\right)$. Hence by Bounded Idealization there exists an element $j \in \mathbb{N}$ such

\footnotetext{
${ }^{23} \mathrm{~A}$ set is bounded if it is an element of a standard set.

${ }^{24} \mathrm{BST}$ was explicitly formulated in [84. Implicitly it is equivalent to the theory of internal sets in some of the theories developed in Hrbacek 74]; see Section 5.4 .
} 
that $i<j$ for all standard $i$; i.e., $j$ is an infinitely large natural number.

\section{Transfer.}

$(\exists x) \Phi(x) \equiv\left(\exists^{\text {st }} x\right) \Phi(x)$

where $\Phi(x)$ is any formula in the $\in$-language, with standard parameters.

An equivalent version of Transfer is that any statement $\Phi\left(a_{1}, \ldots, a_{n}\right)$ (in the $\in$-language) about standard sets $a_{1}, \ldots, a_{n}$ is true when interpreted in the standard universe if and only if it is true when interpreted in the internal universe. This is a modern version of Leibniz's Law of Continuity; see Section 3.1 .

Standardization. For each formula $\Phi(z)$ in the $(\mathbf{s t}, \in)$-language, possibly with parameters, we have the following:

$\left(\forall^{\text {st }} X\right)\left(\exists^{\text {st }} Y\right)\left(\forall^{\text {st }} z\right)(z \in Y \equiv z \in X \wedge \Phi(z))$

No axiom of Separation for arbitrary formulas in the $(\mathbf{s t}, \in)$-language is imposed by BST; for example, the collection $\{n \in \mathbb{N}: \mathbf{s t}(n)\}$ is not a set 25 In its place one has the Standardization principle, to the effect that for any property $\Phi(x)$ expressible in the $(\mathbf{s t}, \in)$-language and any standard set $X$ there is a standard set $Y$ that contains exactly those standard elements of $X$ that have the property $\Phi$. (But $Y$ and $\Phi$ do not have to agree on nonstandard elements of $X$.) Two simple but important consequences of the BST axioms, especially of Standardization, are the following:

(1) If $k, n \in \mathbb{N}, n$ is standard, and $k<n$, then $k$ is also standard; and

(2) If $x$ is a finite real number (i.e., $|x|<n$ for some standard $n \in$ $\mathbb{N}$ ), then there is a (unique) standard real number $r$ such that $r \simeq x$ (such an $r$ is called the standard part of $x$ or the shadow of $x)$.

\footnotetext{
${ }^{25}$ More precisely, BST (as well as IST) proves that there is no set $x$ equal to $\mathbb{N}^{\prime}=$ $\{n \in \mathbb{N}: \mathbf{s t}(n)\}$. Entities like $\mathbb{N}^{\prime}$, that is, those defined as $\{x \in X: \Phi(x)\}$, where $X$ is a true set and $\Phi$ is a formula in the $(\in, \mathbf{s t})$-language, are called external sets (or sometimes semisets), and typically they are non-sets in BST and IST, unless st can be eliminated in some fashion.

Yet BST allows for an implicit introduction of external sets by means of a certain coding defined in 90. This coding system involves not only external sets of internal elements like the collection of all standard integers $\mathbb{N}^{\prime}$ above, but also external sets of external sets, etc. This results in the construction of a universe of Hrbacek's external set theory HST, which extends the given BST universe in the same way that the complex numbers extend the real line by means of representation of a number $a+b i$ as a pair $\langle a, b\rangle$ of real numbers $a, b$. See [90, or [92, Chapter 5].
} 
We first prove (2). Assume $|x|<n$ for some standard $n \in \mathbb{N}$. By Standardization, there is a standard set $Y$ such that

$$
\left(\forall^{\text {st }} z\right)(z \in Y \equiv z \in \mathbb{R} \wedge z<x) .
$$

Note that $Y \neq \emptyset$ ( since $-n \in Y$ ) and $Y$ is bounded above by $n$. By completeness of $\mathbb{R}, Y$ has a supremum $r$, and by Transfer, $r$ is standard.

We now show that $x \simeq r$, so $r$ is the standard part of $x$. If not, then $|r-x|>s>0$ for some standard $s$. This means that either $x>r+s$ or $x<r-s$. In the first case, $r+s \in Y$, contradicting $r=\sup Y$. In the second case, $r-s$ is an upper bound on $Y$, again contradicting $r=\sup Y$.

Now we show that (1) follows from (2). Since $k$ is assumed to be finite, it has a standard part $r$. Then $k$ is the unique integer in the standard interval $[r-0.5, r+0.5)$, so $k$ is standard by Transfer.

We note that Boundedness is a single axiom while Bounded Idealization, Standardization, and Transfer are axiom schemata, that is, they apply to an arbitrary formula $\Phi$ (of a certain type); in this they resemble the schemata of Separation and Replacement of ZFC.

The schemata of Standardization and Transfer are common with IST. The schema of Bounded Idealization is weaker than the full Idealization of IST, but the Boundedness axiom makes up for it, adding a more comprehensive control over the interactions between standard and internal sets in the BST set universe than it is possible in IST.

5.3. Connection with ultrafilters. Let us clarify the connection of these axioms with ultrafilters. Working in BST and given an internal set $x$ and a standard ultrafilter $U$, we say that $x$ is in the monad of $U$ if $x \in A$ for all standard $A \in U$.

Note that, for $x$ in the monad of $U, x$ is nonstandard if and only if $U$ is nonprincipal.

From Boundedness and Standardization it follows that for every $x$ there is a standard ultrafilter $U$ such that $x$ is in the monad of $U$.

From Bounded Idealization it follows that for every standard ultrafilter $U$ there is an $x$ such that $x$ is in the monad of $U$. This is a version of saturation.

In fact, for somewhat stronger versions of these statements (the Back and Forth properties, Hrbacek [79, Section 5]), the implications can be replaced by equivalences.

As a consequence, the axiom schemata of BST can actually be replaced by single axioms. Thus BST is finitely axiomatizable over ZFC; see Kanovei-Reeken [92, Section 3.2]. 
The connection goes deeper. Assuming that $x$ is in the monad of $U$, where $U$ is a standard ultrafilter on a standard set $I$, we have a mapping that assigns to (the equivalence class modulo $U$ of) each standard function $f$ on $I$ the internal set $f(x)$. This mapping is an isomorphism between the standard ultrapower of $V$ modulo $U$, with the ${ }^{*} \in$-relation, and the class $\{f(x): f$ is standard $\}$ of internal sets, with the $\in$-relation [92, Section 6.1].

5.4. External theories. Two theories proposed in Hrbacek [74] and assigned acronyms HST and NST in Kanovei-Reeken 92 are external set theories. They are formulated in the (int, st, $\in$ )-language, where int is a unary predicate of internality (here $\operatorname{int}(x)$ reads " $x$ is internal"). The internal part of these theories satisfies BST, but they admit also external sets. This makes them more powerful, but also more complex.

The two theories differ in the properties of the universe of all (internal and external) sets. It turns out that this universe cannot satisfy all the axioms of ZFC. Thus, HST allows Replacement (and even its stronger version, Collection), while NST makes available Power Set and Choice.

The theory KST proposed in Kawai [103] extends IST by external sets. Andreev and Gordon developed the nonstandard class theory NCT [3]. Roughly speaking, NCT is to BST what the von NeumannGödel-Bernays set theory is to ZFC; in particular, NCT has standard, internal and external classes.

The work of Nelson and his followers demonstrated that a lot of nonstandard mathematics can be carried out by internal means alone; see Section 6.2. External sets are necessary for some more advanced constructions, such as Loeb measures.

There are also nonstandard set theories that do not fit into our twoway classification of axiomatic approaches that well; we mention only the $\alpha$-theory proposed in Benci-Di Nasso [18] and the framework for nostandard analysis developed in ZFC with the axiom of Foundation replaced by the axiom of Superuniversality, by Ballard and Hrbacek [11.

\subsection{Consistency, conservativity and intuitive interpretation.}

Each of these theories is known to be an equiconsistent and conservative extension of ZFC. Here conservativity means that any $\in$-sentence provable in the nonstandard theory is already provable in ZFC; the converse is clearly true as well.

Therefore one might expect that each model of ZFC can be embedded, as the class of all standard sets, into a model of the nonstandard theory. However, this is false for IST; see Kanovei-Reeken [92, 4.5]. In fact ZFC has to be strengthened, for instance by adding the global choice axiom and the truth predicate for $\in$-formulas, in order to be 
able to embed its model, as the class of standard sets, into a model of IST; see [92, 4.6]. For NST this expectation is also false, but it is true for BST as well as for HST.

We pointed out already that an ultrapower of $V$ is non-well-founded, and hence in ZFC there is no mapping of the ultrapower of $V$ onto some transitive class that would convert the membership relation ${ }^{*} \in$ in the ultrapower into a true $\in$-relation. Briefly, the membership relation in the ultrapower is not the actual membership relation $\in$, and cannot be converted to it in the framework of ZFC. Since nonstandard set theories axiomatize ultrapowers or iterated ultrapowers, they transcend ZFC; an intuitive picture of the universe of such a theory properly extends the familiar ZFC universe $V$.

A question then arises to determine what the place of $V$ is in this larger picture. In a discussion of this issue, we have to distinguish between two meanings of the word standard. On one hand, there is the technical meaning; standard objects are the objects in the scope of the predicate st. On the other hand, the expression standard objects is sometimes used to refer to the objects that cousin Georg and many traditional mathematicians are familiar with as the objects from the ZFC universe $V$. These two meanings do not necessarily coincide; in order to perform a disambiguation, we will refer to the standard objects in this second sense as the familiar objects.

5.6. The three pictures. At this point, there are several choices. One can identify the internal numbers and sets with the familiar numbers and sets. Standard reals and sets (i.e., those in the scope of st) are on this view only some of the mathematical entities with which we are familiar, singled out for special attention. One can call this view the internal picture. It is the view adopted in Nelson [127]. We discuss it in detail in Section 7.2 .

However, one can equally well regard the standard numbers and sets as the familiar numbers and sets, with the understanding that standard sets may contain also (what are referred today as) nonstandard elements, or (as Leibniz might have called them) ideal elements or useful fictions; see Section 7.5. One can call this view the standard picture. It is proposed in Hrbacek [75]. In the superstructure framework, it would correspond to viewing the standard copies as the familiar objects. Analogously, in projective geometry an affine plane is outfitted with an assortment of ideal points at infinity; see Section 3.1 . 
External set theories admit yet another picture, the external picture. In these theories one can single out the class of well-founded sets 26 These are generally external. There is a one-one $\in$-preserving mapping $*$ of the well-founded sets onto the standard sets. One then has the option of regarding the well-founded sets as the intuitively familiar objects, and everything else as ideal. In this view, mathematics can be developed in a way similar to the superstructure framework, with the universe of all well-founded sets in place of $V_{\omega}(\mathbb{R})$. This picture was outlined in an appendix to Hrbacek [75] and is fully implemented in Kanovei-Reeken [92].

5.7. The Protozoa metaphor. The following analogy may be helpful in interpreting the three pictures of Section 5.6. Here we are thinking of each real number as an individual (as one does in the superstructure approach). Let us assume we are familiar with the class of animals, and then someone invents the microscope and we discover the protozoa; are we to count them as animals? There are three possible answers.

(1) (External picture) Protozoa are not animals because they fall outside what we previously meant by the word 'animal', but we can invent a new word, 'hyperanimal,' to include animals and protozoa.

(2) (Standard picture) Protozoa are animals and were so all along; there is no change in the meaning of 'animal'. The familiar class of animals contains unfamiliar species like protozoa in addition to the familiar ones. The microscope allows us to see new animals (animals that are new to us, that is).

(3) (Internal picture) Protozoa are animals, and they were familiar all along, even though we weren't aware of them specifically. What is new is our ability to distinguish between microscopic and macroscopic animals: this is what the microscope provides 27

5.8. The intuitive interpretation. The following three points need to be kept in mind.

1. The choice of the nonstandard set theory does not commit one to a particular picture. Nelson used the internal picture, and hence this picture is usually considered an intrinsic part of IST, but IST is equally

\footnotetext{
${ }^{26} \mathrm{~A}$ set $A$ is transitive if any $x \in A$ satisfies $x \subseteq A$. A set $X$ is well-founded if there is a transitive set $A$ such that $X \subseteq A$ and the restriction of the $\in$-relation to $A$ is well-founded. In ZFC all sets are well-founded, but external nonstandard set theories necessarily have also ill-founded sets.

${ }^{27}$ The allusion to Keisler's microscope Keisler 107 is of course intentional.
} 
compatible with the standard picture. The theory and the picture are two separate things. The choice of the picture is not a mathematical issue, since one will get exactly the same mathematical results regardless of the picture, but rather a matter of personal preference.

2. Cousin Georg would likely object to both the internal and the standard picture, on the grounds that numbers and sets as developed around 1872 not long before the 1886 Patent-Motorwagen (see Section 11), i.e., the entities he feels comfortable with, do not fit either in his opinion.

In the case of the internal picture the issue is that, intuitively, every nonempty collection of natural numbers has a least element. Now in a nonstandard set theory like IST there is no set of all nonstandard natural numbers. This may run counter to cousin Georg's expectations concerning the properties of the familiar numbers along the lines of the CD+II mindset (see Section 2.2), where every definite property of natural numbers should necessarily determine a set. Note that st is treated as a definite property in nonstandard set theory: for each $x$, either $\mathbf{s t}(x)$ or $\neg \mathbf{s t}(x)$.

In intuitive terms, we can consider st to be an indefinite, vague property like heap in the paradox of sorites. This indefiniteness is reflected in the formal theory by the nonexistence of the corresponding set. The resistance to this idea is due to an inherent tendency to abstract (form collections).

Cousin Georg's objection to the standard picture is that the familiar set $\mathbb{N}$ just does not contain any ideal elements. A counter-argument is that one can think as if it did. The problems connected with collections of ideal natural numbers without the least element are also perhaps less pressing in this picture. (A personal anecdote: The recent book Hrbacek-Lessman-O'Donovan [81] was first written in the internal picture, which was strongly objected to by some of the referees-perhaps cousin Georg among them. Eventually a switch to the standard picture was implemented, and the book was accepted immediately afterward.)

3. The external picture seems to be the least objectionable from the point of view of cousin Georg (in fact, there seems to be no obvious reason why it should be objectionable at all), but the necessity to work with external sets (in addition to the standard and internal ones) complicates the framework.

A discussion of these various pictures can be found in Hrbacek [77.

5.9. Relative standardness. Arguably, there is not much current mathematical work in nonstandard analysis that actually uses more than one level of standardness. In the superstructure framework, we 
know only of an early paper Molchanov [126], employing two levels. Perhaps one reason for this paucity is that the model-theoretic framework with more than one level of standardness quickly becomes unmanageable because it naturally involves complex combinations of ultrapowers to take care of all the details.

As for the axiomatic framework, there are two distinct approaches.

The first approach is to define different levels or degrees of standardness within a given nonstandard universe of discourse, e.g., a universe satisfying Nelson's internal set theory IST or its BST (bounded set theory) version; see Section 5.1 and Section 5.2.

There are several meaningful approaches to defining relative standardness in this setting. Among them are the following three:

(1) (most natural but not most useful) A set $y$ is standard with respect to $x$ if there is a standard map $f$ such that $x \in \operatorname{dom} f$ and $y=f(x)$; see e.g., Gordon [53].

(2) (following [53]) A set $y$ is standard with respect to $x$ if there is a standard map $f$ such that $x \in \operatorname{dom} f$, all values of $f$ are finite set:28 and $y \in f(x)$.

(3) (following Kanovei [84, Section 3], based on earlier ideas of Luxemburg [121] and Hrbacek). If $\kappa$ is a standard cardinal then one defines sets of order $\kappa$ as those belonging to standard sets of cardinality $\kappa$ or less.

These and similar definitions lead to the internal subuniverses as discussed in Kanovei-Reeken [92], that is, classes $I$ of internal sets satisfying the following: if $x_{1}, \ldots, x_{n} \in I$, where $n$ is a standard number, $f$ is a standard function, and the string $\left\langle x_{1}, \ldots, x_{n}\right\rangle$ belongs to dom $f$ then $f\left(x_{1}, \ldots, x_{n}\right) \in I$. Each such an internal subuniverse $I$ can be considered as a new degree of standardness, that is, the class of all sets standard in the new sense.

Chapter 6 in [92] is devoted to these notions and contains several results related to properties of these generalized notions of standardness.

The second approach to relative standardness is to introduce this complex notion axiomatically, rather than explicitly defining it in a given nonstandard universe. The advantage of this approach is that all the axioms of BST can be relativized to each level of standardness.

The idea to treat levels of standardness axiomatically was proposed by Wallet (see Péraire and Wallet [134]) and fully developed by Péraire [132. Péraire's axiomatic theory RIST is an extension of IST to many levels of standardness. Besides $\in$, its language contains a binary relative

\footnotetext{
${ }^{28}$ Finiteness in IST corresponds to hyperfiniteness in the model-theoretic approach.
} 
standardness predicate $\sqsubseteq$; one can read $x \sqsubseteq y$ as " $x$ is standard relative to $y$." Thus the class $\{x: x \sqsubseteq y\}$ is the level of standardness determined by $y$. The number 0 (or any other object definable without parameters) determines the coarsest level of standardness, which can be identified with the standard sets of IST.

In RIST one can have a finite sequence of numbers, for example $\eta_{0}, \eta_{1}, \eta_{2}$, where $\eta_{0}$ is infinitesimal and each of the other terms is infinitesimal relative to the level of standardness determined by the preceding one. Tao writes:

Having this hierarchy of infinitesimals, each one of which is guaranteed to be infinitesimally small compared to any quantity formed from the preceding ones, is quite useful: it lets one avoid having to explicitly write a lot of epsilon-management phrases such as "Let $\eta_{2}$ be a small number (depending on $\eta_{0}$ and $\eta_{1}$ ) to be chosen later" and "... assuming $\eta_{2}$ was chosen sufficiently small depending on $\eta_{0}$ and $\eta_{1} "$, which are very frequent in hard analysis literature, particularly for complex arguments which involve more than one very small or very large quantity. Tao [158, p. 55]

While it is straightforward to iterate levels of standardness countably many times, or even along any a priori given linear ordering, it is harder to produce satisfactory frameworks that have sequences of levels of standardness of arbitrary hyperfinite length. This is done in Hrbacek [76] and 79]. In such a framework (the theory GRIST) one can have a hyperfinite sequence of natural numbers where each term is nonstandard relative to the previous one. Tao speculated that this feature might be useful in the proof of Szemerédi's theorem, but the only use of it thus far seems to be a characterization of higher-order differentiability in Hrbacek [78. See also [161] for a use of the language of nonstandard analysis in order to avoid a large number of iterative arguments to manage a large hierarchy of parameters.

The axiomatic framework is used in several papers of Péraire (see e.g., [133]), as well as Gordon [54], the more recent work of Di Nasso 
[39], and in [81] (these works use infinitely many levels of standardness) 29 The axiomatic framework hugely simplifies the presentation.

While RIST and GRIST are internal theories, there are also external theories with many levels such as SNST by Fletcher [46] and EST by Ballard [10].

5.10. Constructive aspects of Robinson's framework. The discussion in some of the earlier sections may have given an impression that nonstandard analysis depends on ultraproducts or some similar model-theoretic techniques, and therefore is essentially non-effective. Errett Bishop and Alain Connes 30 have both claimed that nonstandard analysis is somehow fundamentally non-constructive and non-effective. These claims are fundamentally flawed and have been debunked as follows.

5.10.1. Abstract analysis. Methods of nonstandard analysis are often applied in areas that are inherently non-constructive, such as general topology, the theory of Banach spaces, or Loeb measures; we refer to them here as abstract analysis. The nonstandard settings that one needs for abstract analysis are of course also non-constructive, and do imply the existence of ultrafilters. For example, in $\mathrm{IST}^{-}$, a theory obtained from IST by deleting the Axiom of Choice, one can prove the Boolean Prime Ideal Theorem (Every filter can be extended to an ultrafilter) 31 However, the approach not involving nonstandard analysis (if it exists) is equally non-constructive. A detailed example of this phenomenon in the work of Connes may be found in [88].

5.10.2. Nonstandard Peano Arithmetic theories. Classical mathematical results generally do not require the full power of set theory, whether standard or nonstandard. Let PA be (the axiomatic theory of) the Peano arithmetic, which naturally axiomatizes the natural numbers $\mathbb{N}$, and, for each $n$, let $\mathrm{PA}_{n}$ be the $n$-th Peano arithmetic, a theory which

\footnotetext{
${ }^{29}$ In the simplest case one has a nested chain of classes $\mathbb{N}^{s t_{0}} \subseteq \mathbb{N}^{s t_{1}} \subseteq \mathbb{N}^{s t_{2}} \subseteq$ $\cdots \subseteq \mathbb{N}$, where $\mathbb{N}^{s t_{0}}=\mathbb{N}^{s t}$ can be identified with the standard natural numbers and $\mathbb{N}$ is the set of all natural numbers. Thus the higher the $n$, the less standard the integers of $\mathbb{N}^{s t}$ are. In fact the ordering of the levels of standardness does not have to be of type $\omega$. It can be more complicated, have infinite descending chains, even be dense, but it has to have a least element $\mathbb{N}^{s t_{0}}$.

${ }^{30}$ See the references in note 5 .

${ }^{31}$ It may be interesting to note that one cannot prove the full Axiom of Choice; so even the nonstandard abstract analysis, to the extent it is supported by $\mathrm{IST}^{-}$, is actually more "constructive" than the standard abstract analysis carried out in ZFC. See Hrbacek [80] and Albeverio et al. [1, p. 31] for a related discussion.
} 
naturally axiomatizes the structure which contains the set of natural numbers $\mathbb{N}$ along with its consecutive power sets $\mathcal{P}(\mathbb{N}), \mathcal{P}_{2}(\mathbb{N})=$ $\mathcal{P}(\mathcal{P}(\mathbb{N})), \ldots, \mathcal{P}_{n-1}(\mathbb{N})$, so that PA itself is $\mathrm{PA}_{1}$.

The ideas similar to those described for $\mathrm{ZFC}$, can also be applied to define a nonstandard version ${ }^{*} \mathrm{PA}_{n}$ of each $\mathrm{PA}_{n}$. The relations among these theories (between standard and nonstandard versions for the identical and different values of $n$ ) were thoroughfully studied in Henson-Keisler [70] and some related papers. In particular, it was established that each ${ }^{*} \mathrm{PA}_{n}$ is comparable in terms of its strength rather with the standard theory $\mathrm{PA}_{n+1}$ than with its direct standard base $\mathrm{PA}_{n}$.

5.10.3. Constructive nonstandard mathematics. There is extensive work by Palmgren, Avigad [6], Martin-Löf [125], van den Berg et al. [162], on constructive nonstandard mathematics; see Palmgren [131] for a bibliography of the early contributions. These references introduce both syntactic and semantic approaches to nonstandard analysis which are constructive in the sense of Bishop's constructive analysis and MartinLöf's constructive type theory, i.e., based on intuitionistic logic.

5.10.4. Effective content and reverse mathematics. Classical nonstandard analysis actually contains a lot of easily accessible effective content as follows. Sanders [149] establishes that one can algorithmically convert a proof of a theorem in "pure" nonstandard analysis (i.e., formulated solely with nonstandard axioms and nonstandard definitions of continuity, compactness, differentiability, etc.) into a proof of the constructive/effective version of the associated classical theorem.

This work is done in an axiomatic framework (some fragment of full nonstandard set theory) and always produces effective (and even constructive) results when Transfer and Standardization are not used.

The use of the former gives rise to relative computability results in the spirit of Reverse Mathematics, while the use of the latter (or saturation) translates into results computable modulo bar recursion [109]. Osswald and Sanders discuss the constructive content of nonstandard analysis at length in [130], and Sanders [150] discusses how these results undermine the Bishop-Connes critique.

5.10.5. Tennenbaum's theorem. At first glance, even fragments of Robinson's framework based on arithmetic may seem fundamentally nonconstructive from the viewpoint of Tennenbaum's theorem. The theorem literally states that any nonstandard model of Peano Arithmetic is not computable. What this means is that for a nonstandard model $\mathcal{M}$ 
of Peano Arithmetic, the operations $+_{\mathcal{M}}$ and $\times_{\mathcal{M}}$ cannot be computably defined in terms of the operations $+_{\mathbb{N}}$ and $\times_{\mathbb{N}}$ of an intended

model $\mathbb{N}$ of Peano Arithmetic. Similar results exist for fragments; see Kaye [104, § 11.8].

Now, while certain nonstandard models do require non-constructive tools to build, models are not part of Nelson's axiomatic approach IST or its variant BST (see Section [5.2). Furthermore, IST and BST specifically disallow the formation of external sets like "the operation + restricted to the standard numbers." Nelson called attention to this rule on the first page of [127] introducing IST: "We may not use external predicates to define subsets. We call the violation of this rule illegal set formation" (emphasis in original).

Thus, one of the fundamental components of Tennenbaum's theorem, namely the external set "+ restricted to the standard naturals" is missing from the internal set theories IST and BST, as the latter exclusively deal with internal sets. Arguably, therefore, Tennenbaum's theorem is merely an artifice of the model-theoretic approach to nonstandard analysis.

The critique by Connes of Robinson's framework is based on similarly flawed assumptions, namely that the models generally used in Robinson's framework are fundamentally non-constructive and therefore so is nonstandard analysis. It is worth pondering the fact that nonconstructive mathematics is routinely used in physics (see e.g., the discussions of the Hawking-Penrose singularity theorem and the CalabiYau manifolds in [95], undecidability of the spectral gap [34]), without scholars jumping to the conclusion that physical reality is somehow non-constructive.

\section{Physics: Radically elementary modeling}

6.1. Tao on intricate results. Tao wrote in Compactness and contradiction as follows:

The non-standard proofs require a fair amount of general machinery to set up, but conversely, once all the machinery is up and running, the proofs become slightly shorter, and can exploit tools from (standard) infinitary analysis, such as orthogonal projections in Hilbert spaces, or the continuous-pure point decomposition of measures. Tao [159, p. 168]

Here Tao is referring to the fact that if one works from the construction perspective (as he does), then the entire ultrapower construction counts as part of the general machinery. Meanwhile, 
... for particularly intricate and deep results it can happen that non-standard proofs end up being simpler overall than their standard analogues, particularly if the non-standard proof is able to tap the power of some existing mature body of infinitary mathematics (e.g., ergodic theory, measure theory, Hilbert space theory, or topological group theory) which is difficult to directly access in the standard formulation of the argument. [159, p. 169]

Edward Nelson would have likely subscribed to Tao's view as expressed above, but may have added that there is another side of the coin which is what we shall refer to as Radically Elementary Modeling. This term alludes to Nelson's book Radically Elementary Probability Theory [128].

6.2. The physicist's vibrating string. We are interested in developing a mathematical model of a vibrating string. First we will illusrate the viewpoint of a physicist by providing some quotations. In the celebrated Berkeley Physics Course (vol. 3), Frank Crawford writes:

(Sec. 2.1) If a system contains a very large number of moving parts, and if these parts are distributed within a limited region of space, the average distance between neighboring moving parts become very small. As an approximation, one may wish to think of the number of parts as becoming infinite and the distance between neighboring parts as going to zero. One then says that the system behaves as if it were "continuous". Implicit in this point of view is the assumption that the motion of near neighbors is nearly the same. Crawford [33, p. 48]

The next quotation deals with a uniform beaded string having $N$ beads and with fixed ends:

(Sec. 2.2) We now consider the case where $N$ is huge, say $N=1,000,000$ or so. Then for the lowest modes (say the first few thousand), there are very large number of beads between each node. Thus the displacement varies slowly from one bead to the next [We shall not consider here the highest modes, since they approach the "zigzag limit", where a description using a continuous function is not possible.] (ibid., p. 51)

Furthermore,

(Sec. 2.4) In sec. 2.2 we considered a continuous string. . In this section we will find the exact solutions for the 
modes of a uniform beaded string having $N$ beads and with fixed ends. In the limit that we take the numbers of beads $N$ to to be infinite (and maintain the finite length $L$ ), we shall find the standing waves that we studied in Sec. 2.2. Our purpose is not merely that, however. Rather, we shall find that, in going to the limit of a continuous string, we discarded some extremely interesting behavior of the system. (ibid., p. 72)

These comments would be considered by traditionally trained mathematicians as an "informal discourse of a physicist." But for a mathematician trained in nonstandard analysis, they can be easily translated into a perfectly formalized mathematical text.

6.3. The hyperfinite vibrating string. We will now formalize Crawford's approach in Nelson's IST. Let $\Sigma_{N}$ be the following system of differential equations:

$$
\Sigma_{N}\left\{\begin{array}{lr}
\frac{d x_{j}}{d t}=y_{j}(t), & j=1, \ldots, N-1 \\
\frac{d y_{j}}{d t}=\frac{K^{2}}{h^{2}}\left(x_{j-1}(t)-2 x_{j}(t)+x_{j+1}(t)\right), & j=1, \ldots, N-1 \\
x_{0}(t)=x_{N}(t)=0 &
\end{array}\right.
$$

where $N \cdot h=1$.

Assuming $K=1$, from elementary calculus we know that the solutions are given by the following equations:

$$
x_{j}(t)=\Sigma_{n=1}^{N-1} u_{n} \cos \left(\omega_{n} t\right)+\frac{v_{n}}{\omega_{n}} \sin (n \pi j h),
$$

where

$$
\begin{aligned}
& \omega_{n}=\frac{2}{h} \sin \left(\frac{n \pi h}{2}\right) \\
& u_{n}=2 h \Sigma_{k=1}^{N-1} x_{n}(0) \sin (k \pi n h) \\
& v_{n}=2 h \Sigma_{k=1}^{N-1} y_{n}(0) \sin (k \pi n h)
\end{aligned}
$$

In classical mathematics one considers the continuous wave equation:

$$
\Sigma \quad \begin{cases}\frac{\partial^{2} X}{\partial t^{2}}(z, t) & =\frac{\partial^{2} X}{\partial z^{2}}(z, t) \\ X(0, t) & =X(1, t)=0\end{cases}
$$

to be "the model" and proves the existence of solutions $(t, z) \mapsto X(t, z)$, continuous with respect to $t$ and $z$ wich satisfy (6.4); if one is interested in computer simulations it can be proved that suitable solutions of the discrete (6.1) converge in some sense to a solution of (6.4) when $N$ 
tends to infinity. By the way, in classical mathematics, the system (6.1) is usually viewed as an approximation of (6.4).

But in nonstandard analysis we can consider (6.1) to be "the model" provided that $N$ is nonstandard (infinite). Explicit formulas (6.2) still stand. Typically a physicist is less interested in the existence of solutions than in their properties. For instance he might be interested in the "shape" of the string at time $t$, namely, in the aspect of the "dotted line" in $[0,1] \times \mathbb{R}$ :

$$
\left\{\left(n d z, u_{n}(t)\right): n=0,1, \ldots, N\right\}
$$

with $d z=h$. An important question is whether the "dotted line" seems continuous. (The physicist asks "whether the displacement varies slowly from one bead to the next"). The mathematical formalization is the following.

Definition 6.1. The "dotted line" (6.5) is said to be $S$-continuous if:

$$
k d z \approx 0 \Longrightarrow u_{n+k}(t) \approx u_{n}(t) .
$$

The S-continuity of the initial condition $x_{n}(0) ; n=0,1, \cdots, N$ is insufficient to imply the S-continuity of the solution. What is required in addition is that the initial energy

$$
E(0)=\frac{1}{2} \sum_{k=1}^{N-1}\left(\frac{x_{n+1}(0)-x_{n}(0)}{h}\right)^{2} h+\sum_{k=1}^{N-1} y_{n}(0)
$$

be limited. In fact consider an initial condition such that $x_{n}(0)=0$; if an unlimited energy at time $t=0$ is contained in the unlimited modes $\lambda_{n}$ it turns out that after some duration all this energy will concentrate at some point and "break" the solution; for details see Delfini-Lobry [35]. It is one of the interesting aspects of the behavior of the system that we can observe when we do not discard high modes. Notice that in the preceding lines one uses only the Idealization axiom of IST.

If one wishes to make the connection with the continuous model, one uses the shadow of the "dotted line" whose definition uses the full strength of the Standardization axiom. Then it can be proved under suitable asumptions on the initial conditions (including limited energy) that the shadow of the "dotted line" is the graph of a differentiable function $\varphi(t, z)$ which is a solution of (6.4).

We acknowledge that our example is very simple and that physicists did not await Robinson to understand all the information contained in formulas (6.2) and (6.3). 
6.4. Hyperfinite Brownian motion. This point of view was advocated in Nelson [128] (a less elementary approach using Loeb measures was pioneered in Anderson [2]), where Nelson defined the Brownian motion as a discrete random walk of infinitesimal step size at times $0, d t, 2 d t, \ldots, k d t, \ldots, N d t=1$, with $d t$ infinitesimal. Namely, we set

$$
\xi_{0}=0 ; \quad \xi_{t+d t}=\xi_{t}+z_{t} \sqrt{d t}
$$

where $z_{t}$ is a sequence of independent random variables taking the values +1 or -1 with probability $\frac{1}{2}$. The probability space for such a process is just the (hyper)finite set $\Omega=\{-1,+1\}^{N}$ with its $\sigma$-algebra equal to $P(\Omega)$. A trajectory of this Brownian motion is defined as the mapping $t \mapsto x_{t}$ with domain in what Nelson refers to as the nearinterval, namely the hyperfinite set $\{0, d t, 2 d t, \ldots, k d t, \ldots, N d t=1\}$. The important point here is that despite its discrete definition, which fits well with the intuition that "at each instant one chooses at random" (notice that in the limit $d t \rightarrow 0$ this concrete meaning is lost), the notion of continuity is perfectly defined by $S$-continuity (see above). Then Nelson proves the following:

Almost surely, a trajectory of the Brownian motion is S-continuous.

Here almost surely means that for every standard $\varepsilon>0$ the (external) set of trajectories that are not S-continuous is contained in a (true) set of probability less than $\varepsilon$. This result is obtained within a very light subsystem of IST (just Idealization is needed). The title of Radically Elementary Probability Theory delivers on its promise. In fewer than 80 pages, starting from the early beginnings including nonstandard analysis and some basics of probability theory, the mathematical model for the physical Brownian motion is constructed. Moreover the last chapter called The de Moivre-Laplace-Lindeberg-Feller-WienerLévy-Doob-Erdos-Kac-Donsker-Prokhorov theorem provides a large amount of modern results on stochastic processes. It is the decision not to replace clear nonstandard statements by wordier conventional paraphrases that makes things elementary. Let us quote Nelson's Preface, paragraphs 2 and 3:

This work is an attempt to lay new foundations for probability theory, using a tiny bit of nonstandard analysis. The mathematical background required is little more than which is taught in high school, and it is my hope that it will make deep results from the modern theory of stochastic processes readily available to anyone who can add, multiply and reason. 
What makes this possible $i s$ the decision to leave the results in non-standard form. Nonstandard analysts have a new way of thinking about mathematics, and if it is not translated back into conventional terms then it is seen to be remarkably elementary. Nelson [128, p. vii] (emphasis ours)

At the next stage, in order to connect elementary results to classical ones, the full IST system is used to prove the (formal) mathematical equivalence of the hyperfinite model with the classical Wiener process. Let us quote again from Nelson:

The purpose of this appendix is to demonstrate that theorems of the conventional theory of stochastic processes can be derived from their elementary analogues by arguments of the type usually described as generalized nonsense; there is no probabilistic reasoning in this appendix. This shows that the elementary nonstandard theory of stochastic processes can be used to derive conventional results... [128, p. 80]

Nelson goes on to make the following additional point:

... on the other hand, it shows that neither the elaborate machinery of the conventional theory nor the devices from the full theory of nonstandard analysis, needed to prove the equivalence of the elementary results with their conventional forms, add anything of significance: the elementary theory has the same scientific content as the conventional theory. This is intended as a self destructing appendix 32 Nelson [128]

This view regarding stochastic processes was extended to diffusion:

$$
\xi_{0}=0 ; \quad \xi_{t+d t}=\xi_{t}+f\left(\xi_{t}\right) d t+z_{t} \sqrt{d t}
$$

in Benoit [20], to stochastic analysis as applied to finance in van den Berg [164, and to complex physical particle systems in Weisshaupt [170], [171]. In a similar vein, Lobry [115], Lobry-Sari [116] advocate

\footnotetext{
${ }^{32}$ Nelson's cryptic comment calls for a clarification. What Nelson is apparently referring to is the fact that the conventional formalism for random walks in particular and stochastic analysis in general, as developed by Kolmogorov and others, relied on elaborate machinery based on measure theory. Nelson viewed the replacement of elaborate machinery by radically elementary considerations as a positive scientific development, which however has self-destructing aspects as far as the work of the traditional practitioners themselves is concerned.
} 
viewing ODEs with discontinuous right hand side on the basis of a simple Euler scheme with infinitesimal step. The path of Radically Elementary Modeling was also pursued in Diener-Lobry [37], Harthong [66], Fliess [47] and other works.

\section{More ON NELSON}

Bien que tous les mathématiciens ne le reconnaissent pas, il existe une "réalité mathématique archaïque". Comme la réalité du monde extérieur, celle-ci est a priori non organisée, mais résiste à l'exploration et révèle une cohérence. Non matérielle, elle se situe hors de l'espace-temps.

A. Connes [31]

The idea that there is a pure world of mathematical objects (and perhaps other ideal objects) totally divorced from our experience, which somehow exists by itself is obviously inherent nonsense. M. Atiyah [5, p. 38]

We first give a more detailed discussion of the II hypothesis outlined in Section 2.2.

7.1. Kleene, Wang, Putnam, and others on the II. Many set theorists think of the semantics of set theory as given in terms of an intended model or intended interpretation, in accordance with a realist philosophy of mathematics; for details see Maddy [122. Intended interpretations concern not merely the natural numbers but, more generally, purported entities in something described as "a mathematical practitioner's universe," "primordial mathematical reality" (a term coined by A. Connes), or the like. Concerning such II hypotheses, Kleene wrote: 
Since a formal system (usually) results in formalizing portions of existing informal or semiformal mathematics, its symbols, formulas, etc. will have meaning or interpretations in terms of that informal or semiformal mathematics. These meanings together we call the (intended or usual or standard) interpretation or interpretations of the formal system. Kleene [108, p. 200]

In a similar vein, Hao Wang writes:

The originally intended, or standard, interpretation takes the ordinary nonnegative integers $\{0,1,2, \ldots\}$ as the domain, the symbols 0 and 1 as denoting zero and one, and the symbols + and $\cdot$ as standing for ordinary addition and multiplication (see section "Truth definition of the given language" in Wang [169]).

An intended model could be defined as one that "reflects our intuitions [about natural numbers] adequately" Quinon-Zdanowski [138, p. 313] (emphasis added). Haim Gaifman writes:

Intended interpretations are closely related to realistic conceptions of mathematical theories. By subscribing to the standard model of natural numbers, we are committing ourselves to the objective truth or falsity of numbertheoretic statements. ... Realism and intended interpretations are thus intimately related; often they are treated as the same problem. Gaifman [48, p. 15]

Thus the II hypothesis entails that $\mathbb{N}$ obtains a detailed reference in the ordinary counting numbers. Meanwhile, Robinson wrote: "mathematical theories which, allegedly, deal with infinite totalities do not have any detailed ... reference."33 [142, p. 42]

Weber claimed to quote 34 Leopold Kronecker as positing an allegedly immutable status of the integers "whereas everything else is the work of man." Weber's quote is often misattributed to Kronecker himself. However, Kronecker specifically wrote, on the contrary, that numbers were a creation of the human mind, and contrasted numbers that are human artefacts, with space and time which he felt were outside the mind (possibly following the traditional pre-Einsteinian philosophers):

The principal difference between geometry and mechanics on one hand, and the other mathematical disciplines

\footnotetext{
${ }^{33}$ A related point was made by Salanskis; see note 37.

${ }^{34}$ The adage Weber reports in Kronecker's name is known not to appear in any of Kronecker's writings; see Ewald [44, p. 942, note a].
} 
we comprehend under the name of arithmetic, consists according to Gauss in this: the object of the latter, number, is a pure product of our mind, while space as well as time has reality also outside of our mind which we cannot fully prescribe a priori. Kronecker [110, p. 339]

See also Gauthier [49, p. 163]. Hilary Putnam doubted our ability to fix an intended interpretation and seemed to treat the II as a throwback to Kantian noumena [136, p. 482] 35 For responses to Putnam see e.g., Horsten [73], Gaifman [48].

7.2. Predicate on the familiar real line. In the internal view of IST, rather than thinking of the standard sets as being the familiar ones, embedded in a larger nonstandard world, one essentially thinks of the nonstandard universe as the familiar world (in the terminology of Section 5.5), with standard structures being picked out of it by means of a suitable predicate.

On this view, one has the real numbers including both infinite and infinitesimal reals, and one can say when two finite real numbers have the same standard part, etc. In this picture, we think of the familiar real line as what in the other picture would be the nonstandard one, and then we have a predicate on that, which corresponds to the range of the star map in the other approach. So some real numbers are standard, and some functions are standard and so on.

Hamkins wrote: "One sometimes sees this kind of perspective used in arguments of finite combinatorics, where one casually considers the case of an infinite integer or an infinitesimal rational." That kind of talk may seem alien to cousin Georg (see Section 1), but for those who adopt the picture it is useful. In a sense, one goes the whole nine yards into the nonstandard realm until it turns familiar.

There are several characterisations of Nelson's framework. Each characterisation employs a slightly different philosophical starting point. Peter Loeb's position as expressed in [118, p. vii] is that (1) Nelson is working exclusively in the nonstandard universe, and (2) there is no standard world in this setting. This second point is of course mathematically correct to the extent that the predicate st violates the axiom

\footnotetext{
${ }^{35}$ Putnam's comment there about "noumenal waifs" indicates an impatience with the typical post-Dedekind mathematician's assumption that $\mathbb{N}$ finds a detailed reference in the ordinary intuitive counting numbers. Putnam seems to view the latter as inaccessible noumena, the mathematician's identification of $\mathbb{N}$ with these noumena as an unwarranted assumption, and the search for the intended interpretation as a futile search for parenthood for the said waiflike noumena. See further in note 37.
} 
of separation. As far as the first point is concerned, it involves a bit of an equivocation on the meaning of the word standard:

(a) its technical meaning in the context of Robinson/Nelson, and

(b) the meaning of ordinary/usual.

The equivocation is disambiguated in Section 5.5. In line with the CD+II mindset (see Section 2.2), cousin Georg (see Section 11) would bridle at the idea that the ordinary/usual real line should contain infinitesimals in any sense, and so Loeb is correct in this sense, as well. Other experts in nonstandard analysis point out a complementary mathematical point that is also valid, namely the following.

Nelson demonstrated that infinitesimals can be found within the ordinary real line itself in the following sense. Infinitesimals are found in the real line by means of enriching the language through the introduction of a unary predicate st and postulating an axiom schema (of Idealization), one of most immediate instances of which implies the existence of infinitely large integers and hence nonzero infinitesimals, as in his 1977 article; see Katz-Kutateladze [97] for a related discussion. Recall that Nelson's framework Internal Set Theory (IST) is a conservative extension of ZFC. In other words, the entire package goes over, including each article published in the Annals of Mathematics so long as it makes no use of Sarah's switch (see Section 1) st.

To put it more colorfully, infinitesimals were there all along, but cousin Georg hasn't noticed them.

7.3. Multiverse. Hamkins [61], 62] proposes a view of the foundations of mathematics where there are many distinct concepts of set, each instantiated in the corresponding set-theoretic universe These works formulate a number of principles that the multiverse should satisfy. An interesting observation for our purposes is that the following principle is compatible with Hamkins's multiverse.

Principle B: For every ZFC universe $V$ in the multiverse there is a class $S \subseteq V$ such that all the axioms of the theory BST hold in $(V, \in, S)$.

In other words, given any universe $V$ and a class $S$ as above, the predicate $\mathbf{s t}=\mathbf{s t}_{V, S}$ defined by

$$
\operatorname{st}(x) \text { if and only if } x \in S
$$

distinguishes some elements of $V$ as standard, in a way that makes BST hold, without adding any new elements to $V$. Thus Nelson's claim that the infinitesimals have been there all along without being noticed (see Section 7.2) is literally true in this framework, though in the context of BST rather than Nelson's original theory IST. For the 
benefit of readers familiar with model theory and BST, we give some technical underpinnings of these claims. Victoria Gitman and Hamkins construct a "toy" model of the multiverse axioms in ZFC; see [50. We show that Principle B holds in this model.

Theorem 7.1. The Gitman-Hamkins model satisfies the Principle B.

Outline of proof. One can start with the fact that a standard core interpretation for BST is definable in ZFC (Kanovei-Reeken [92, Theorems 4.1.10(i) and 4.3.13]), where the expression "standard core" indicates that the universe $V$ of $\mathrm{ZFC}$ is isomorphic to the universe of standard sets in the interpretation.

An immediate corollary [92, Corollary 4.3.14] is that for every model $\left(M, \in_{M}\right)$ of ZFC there is a corresponding model $\left(N, \in_{N}, S_{N}\right)$ of BST, in which the class of standard sets $S_{N}$ is isomorphic to $M$.

The multiverse of Gitman and Hamkins consists of all countable computably saturated models of ZFC. It is easy to see that if $\left(M, \in_{M}\right)$ is countable and computably saturated, then also the corresponding $\left(N, \in_{N}, S_{N}\right)$ is countable computably saturated. Furthermore, $\left(N, \in_{N}\right)$ and $\left(M, \in_{M}\right)$ have the same theory and the same standard system. Hence they are isomorphic [50, Key Lemma 6]. If $S$ is the image of $S_{N}$ under such an isomorphism, then $\left(N, \in_{N}, S_{N}\right)$ and $\left(M, \in_{M}, S\right)$ are also isomorphic. In particular, $\left(M, \in_{M}, S\right)$ satisfies BST.

It is not known whether BST can be replaced by Nelson's IST in this argument; see the related discussion in Section 5.5. Essentially the same argument works for the theory GRIST (see Section 5.9) starting with the fact that a standard core interpretation for GRIST is definable in ZFC, Hrbacek [80].

Theorem 7.2. One can consistently assume that every universe of $\mathrm{ZFC}$ in the multiverse can be stratified into many levels of relative standardness by a binary relation $\sqsubseteq$ in such a way that all the axioms of GRIST hold in $(V, \in, \sqsubseteq)$.

7.4. Switches. Hamkins 63, 64] discusses the concept of switches, exemplified by the continuum hypothesis $(\mathrm{CH})$. Here the terminology of switch implies that it can be turned on or off at will. Similarly, using modern techniques of forcing, one can pass from a model of ZFC that satisfies $\mathrm{CH}$ to one that satisfies $\neg \mathrm{CH}$ and vice versa.

The availability of the predicate st in the language is also a type of switch. Namely, all models of ZFC can be built up into models of BST, turning the switch on as it were. Meanwhile, the forgetful functor (just forget about the switch in the glove compartment) takes you from BST back to ZFC. 
The analogy with switches is that one model has infinitesimals, while the other doesn't. This is analogous to one model possessing a set of intermediate cardinality between $\mathbb{N}$ and $\mathbb{N}^{\mathbb{N}}$, and the other not. Both switches arguably challenge the II hypothesis; see Section 2.2.

Now st is a different type of switch, but whatever the situation may be for the ontology and epistemology of switches, Sarah's switch implies (not merely that one can fly faster than drive but) that the discovery of the switch leaves all theorems in place, so that Nelson's real numbers can be viewed as the familiar ones, and the infinitesimals have been there all along without being noticed-even though the familiar model from the last third of the 19th century apparently didn't have them.

7.5. Historical antecedents. The view that numbers come in more than one flavor is closely parallel to the dichotomy of assignable vs inassignable quantities (the latter being viewed as useful fictions) in Leibnizian calculus; see Bair et al. [8] and Bascelli et al. [16] for more details. Even earlier, Fermat's technique of adequality exploited procedures using $E$ in a striking anticipation of later infinitesimal techniques; see [100]. Meanwhile L. Carnot spoke of quantités désignées and quantités auxiliaires in 1797; see Barreau [12, p. 46, 53].

Stolz, du Bois-Reymond, and others were working on infinitesimals at the end of the 19th century, and had they joined forces with Frege or Peano to conceive an axiomatisation that would actually incorporate Leibniz's assignable vs inassignable distinction, 20th century mathematics may have looked different. This viewpoint involves being able to conceive of the history of mathematics as something other than inevitable march toward Weierstrassian epsilontics and Cantor-Dedekind reals. In more precise philosophical terms, it involves envisioning the history of mathematics in terms of the Latin model rather than the butterfly model, to borrow the terminology from Ian Hacking [59].

To comment on Hacking's distinction between the butterfly model and the Latin model, we note the contrast between a model of a deterministic biological development of animals like butterflies, as opposed to a model of a contingent historical evolution of languages like Latin.

Hacking's dichotomy applies to the development of the field of mathematics as a whole. Some scholars view the development of mathematics as a type of organic process predetermined genetically from the start, even though the evolution of the field may undergo apparently sudden and dramatic changes, like the development of a butterfly which passes via a cocoon stage which is entirely unlike what it is pre-destined to produce. 
The Latin model acknowledges contingent factors in the development of an exact science (mathematics included), and envisions the possibility of other paths of development that may have been followed. For example, had an axiomatic formalisation of infinitesimals been proposed earlier, it might have been incorporated into the early formalisations of set theory, and spared us the inanity of the Cantor-Russell anti-infinitesimal vitriol, reflecting the state of affairs in mathematical foundations during the second half of the 19th century; for additional details see [102].

Hacking's perspective is at odds with some of the received history of mathematical analysis. A related point is made by P. Mancosu in the following terms:

the literature on infinity is replete with such 'Whig' history. Praise and blame are passed depending on whether or not an author might have anticipated Cantor and naturally this leads to a completely anachronistic reading of many of the medieval and later contributions. Mancosu [123, p. 626]

In his critique of intuitionism, Bernays introduced a distinction related to assignable/inassignable in terms of accessible vs inaccessible:

Brouwer appeals to intuition, but one can doubt that the evidence for it really is intuitive. Isn't this rather an application of the general method of analogy, consisting in extending to inaccessible numbers the relations which we can concretely verify for accessible numbers? As a matter of fact, the reason for applying this analogy is strengthened by the fact that there is no precise boundary between the numbers which are accessible and those which are not. Bernays [21] (translation by Charles Parsons)

Thus, we obtain infinitesimals as soon as we assume that (1) there are assignable/standard real numbers, that obey the same rules as all the real numbers, and (2) there are real numbers that are not assignable. Of course, neither Leibniz nor Carnot employed any set-theoretic notions to specify an ontology of their infinitesimals, but their procedures find better proxies in modern infinitesimal frameworks than in modern Weierstrassian ones 36 In more technical terms, one considers the

\footnotetext{
${ }^{36}$ The procedures vs ontology distinction is dealt with in greater detail in the articles Borovik-Katz [28, Bair et al. [7, Bascelli et al. [16], Błaszczyk et al. [24, Błaszczyk et al. [25].
} 
ordinary ZFC formulated in first order logic, and adds to it the unary predicate st and the axiom schemata.

Robinson's framework has enabled a reappraisal of the procedures of the pioneers of infinitesimal analysis. For a broad outline of such a program see the studies [8] (2013), [27] (2013), [15] (2014), 25] (2017). Specific scholars studied include

- Stevin in [96] (2012);

- Gregory in [17];

- Fermat in [100 (2013);

- Leibniz in [101] (2012), [102] (2013), [153] (2014), [16] (2016), 24] (2017);

- Euler in [87] (2015), 77] (2017);

- Cauchy in (Laugwitz [111]) and in [94] (2011), 28] (2012).

\section{ReEB, NAIVE Integers, And $\operatorname{Claim} Q$}

Georges Reeb's position regarding the use of infinitesimals was developed in his essay entitled La mathématique non standard vieille de soixante ans? There are two distinct versions of the essay, [139] (1979) and [140] (1981). The 1979 version of the essay was reprinted in Salanskis [148]. The book gives an account of the constructivist view of Reeb. Reeb was closely associated with J. Harthong in his philosophy of mathematics. They published the article Intuitionnisme 84 [67] in the book Barreau-Harthong [14]. One can distinguish at least three distinct approaches to the problem of motivating a non-naive integer in Reeb, as detailed in the next three sections. See also Lobry [114, Diener-Diener [36, p. 4].

8.1. Nonformalizable intuitions. Reeb's position concerning naive integers can perhaps be described as follows. The naive integers are those integers that all members of humanity share before they understand any advanced mathematics. Such naive integers are already present in any formal language since language is necessarily a succession of symbols, rather than an unordered collection of symbols.

If one accepts a formal language (e.g., that of $\mathrm{ZF}$ ) with respect to which $\mathbb{N}$ is defined, one must accept also that one understands the following informal reasoning:

- We recognize " 1 " of $\mathbb{N}$ as the naive one;

- We recognize " 2 " of $\mathbb{N}$ as the naive two;

- etc.

- If we recognize " $n$ " of $\mathbb{N}$ as the naive $\mathbf{n}$ then we recognize " $n+1$ " of $\mathbb{N}$ as the naive $\mathbf{n}$ plus one. 
Reeb then argues that an assertion to the effect that every element of $\mathbb{N}$ is naive is not supported by any formal mathematics, and arrives at his

"Claim Q": The naive integers don't fill up $\mathbb{N} 37$

Thus claim $\mathrm{Q}$ is at tension with the CD+II mindset (see Section 2.2).

To anyone familiar with model theory, Reeb's "claim Q" could easily be interpreted in terms of the existence of nonstandard models of the natural numbers, whether in PA or ZF, first constructed in Skolem [154]. However, Reeb [139] takes a more "fundamental" attitude and seems to argue for his "claim Q" somehow from first principles, the naive integers being taken to be available before a commitment to formal mathematics. Reeb comments that being naive is not a mathematical concept, hence not formalizable; hence they cannot be said to fill up $\mathbb{N}$.

8.2. Ideal intruders. In [140], Reeb takes a different tack, and argues that any infinitary construction in mathematics, as recognized at least since Hilbert [72], necessarily introduces ideal elements not intended by initial naive intuitions:

D. Hilbert - pour nous en tenir à un seul nom - a montré clairement, dans son célèbre article sur l'infini, comment la formalisation (par exemple $\mathbb{N}, \mathbb{R}, \ldots$ ) de notions concrètes (les entiers de tout le monde, les points du continu intuitifs) introduit nécessairement, en quelque sorte contre la vigilance du formalisateur d'abondants objets idéaux, non désirés. Reeb [140, p. 149]

Reeb goes on to refer to such elements as "des intrus idéaux inévitables lors de la formalisation."

8.3. Link between intuitionism and nonstandard analysis. In the book Diener-Reeb [38, Chapter 9], Reeb provides yet another account of a non-naive integer in an intuitionistic setting, in terms of the size of a hypothetical solution to $x^{n}+y^{n}=z^{n}$ (this was before A. Wiles; of course Fermat's last theorem can be replaced by a conjecture that is still open).

\footnotetext{
${ }^{37}$ Reeb's term in the original French was Constat $Q$ which we loosely translate as Claim $Q$ fully aware of the inadequacies of such a translation. The difficulty of the term was analyzed by Salanskis, who noted: "Le problème du remplissement de $\mathbb{N}$ par les naifs est totalement dénué de sens si l'on ne joue pas le jeu de rêver que les formalismes suscitent des référents." Salanskis [147] (translation: "The problem of filling $\mathbb{N}$ by the naive integers is totally meaningless if one is not playing the game of dreaming that formalisms generate referents.")
} 
Surprising as it may seem to the uninitiated, the French school of nonstandard analysis draws a direct connection between Brouwer's intuitionism and nonstandard analysis. We refer to Diener-Reeb [38, Chapter 9] and Harthong-Reeb [67] for a detailed discussion, while we sketch the motivation for this connection as follows.

L. E. J. Brouwer was the founder of intuitionism, the first school of constructive mathematics; the latter aims to provide a computational foundation for mathematics based on the Brouwer-HeytingKolmogorov (BHK) interpretation of logic.

To identify a part of classical mathematics as not-acceptable in intuitionistic mathematics, Brouwer introduced a technique which would later be called "Brouwerian counterexamples;" see Mandelkern [124] for an overview.

Such counterexamples come in strong and weak flavors and are meant to cast doubt on the constructive/intuitionistic acceptability of a given theorem or axiom. A weak Brouwerian counterexample against the law of excluded middle (LEM) is as follows:

$A \vee \neg A$ is not acceptable (under the BHK interpretation) because there is no algorithm to decide whether $A=$ 'Goldbach's conjecture' is false or not (or any asyet unproved conjecture).

However, Goldbach's conjecture has been verified using computers up to very large numbers. Thus, there is the possibility, discussed at length in [67], that Goldbach's conjecture is false, but that the counterexample cannot be constructed (in principle and/or in practice). Hence, Goldbach's conjecture would be "true in the real world", but false in principle. Here, the real world could be either "the physical world" or the world of constructive mathematics, according to [67].

In other words, all the natural numbers one can construct (in principle or in practice) do satisfy Goldbach's conjecture, but "there are out there" numbers which do not. Following [67], one could refer to the former (constructive) numbers as naive integers (satisfying Goldbach's conjecture) and to the others as non-naive. Now, Goldbach's conjecture is just one example, and there will always be unsolved conjectures, so the previous idea is persistent in that sense. The above reasoning is how one could interpret the adage by Reeb and Harthong that "the naive integers do not fill up $\mathbb{N}$ " based on Brouwer's weak counterexamples to LEM.

Meanwhile, the observation that "the naive integers do not fill up $\mathbb{N}$ " is the basic motivation for having nonstandard numbers in [67], and we 
observe how Brouwer's intuitionism motivates the existence of nonstandard numbers, especially in the sense of Nelson's IST.

8.4. Nelson-style motivations. There are Nelson-style motivations for such a claim $Q$ but they don't seem to be the same as Reeb's. There are various motivations for why naive integers shouldn't exhaust $\mathbb{N}$. One of them is in terms of a multitude that's too vast to be expressed by even a computer the size of the universe running the entire time allotted to our civilisation and exploiting the fastest growing functions in our logical arsenal including superbusy beavers. Such a number could function as infinite for all practical purposes at the naive level. This indicates a useful lack of homogeneity of $\mathbb{N}$ and a promise of a richer structure which is better captured in terms of an enriched syntax as in Nelson's system, which singles out standard (or assignable) elements out of $\mathbb{N}$ by means of a single-place predicate violating the separation axiom.

8.5. Quantum intuitions. The breakdown of infinite divisibility at quantum scales poses an undeniable challenge to the CD+II mentality (see Section 2.2). It makes physically irrelevant a literal reading of the mathematical definition of the derivative in terms of limits as $\Delta x$ tends to zero, since attempting to calculate the derivative for increments below the Planck scale would yield physically meaningless results. Rather, quotients like $\frac{\Delta y}{\Delta x}$ need to be taken in a certain range, or at a suitable level. Attempts to formalize Planck's $\hbar$ as an infinitesimal go back at least to Harthong [66], Werner-Wolff [172]. The article Nowik-Katz [129] $]^{38}$ developed a general framework for differential geometry at level $\lambda$. In the technical implementation $\lambda$ is an infinitesimal but the formalism is a better mathematical proxy for a situation where infinite divisibility fails for physical reasons, and a scale for calculations needs to be fixed accordingly.

If one accepts Reeb's "Claim Q," then any non-naive integer can be described as infinite in the sense that it is greater than any naive integer. Once one forms the field of fractions, the inverse of such an infinite integer in this sense becomes an infinitesimal.

\footnotetext{
${ }^{38}$ In his technical report for the CNRS in 1985, Alain Connes wrote: "La séduction de l'analyse non standard est due en grande partie à la création d'un vocabulaire suggestif; la géométrie différentielle s'est bien gardée de céder à cette tentation, etc." (cited in Barreau [13, p. 35]). Had we been aware of Alain Connes's seductive comment at the time we might have subtitled our article [129] "Differential geometry reduced at last."
} 


\section{Conclusion}

While mathematicians may not always think of set theory as being dynamic, such a switch in foundational thinking is helpful in appreciating the advantages of the framework developed by Robinson, Nelson, and others.

Just as the automobiles have gotten better over the past 130 years, so also there is room for improvement as far as the 1870 set-theoretic foundations are concerned. The automobile industry is dynamic and flexible willy-nilly (if you don't innovate, your competitors will) but the received, and outdated, views on set theory seem to have cornered the market like an intended monopoly, alluded to in Section 7.1. Automobiles today have numerous options, from fuel injection, 4-wheel drive, convertible, and GPS to more futuristic ones like lift-off abilities to various degrees, with broad agreement as to the general utility of such options some of which have become part of the standard package. We argue in favor of inclusion of the infinitesimal option as part of the core package of set-theoretic foundations.

A natural place to start would be in education, so as to restore infinitesimals to the calculus curriculum, as in Cauchy's classroom at the Ecole Polytechnique. Accordingly, over the past few years we have trained over 400 freshmen using Keisler's infinitesimal calculus textbook [107], and summarized the results in the study Katz-Polev [99]. At the high school level infinitesimal calculus has been taught in Geneva for the past twelve years, based on the approach developed in [81].

In addition to serving as a fruitful tool for what Leibniz called the Ars inveniendi, Robinson's framework has occasioned a deepened reflection on mathematical foundations in general and the meaning of number in particular.

\section{ACKNOWLEDGMENTS}

We are grateful to Eric Leichtnam and Dalibor Pražák for helpful comments on an earlier version of the manuscript. M. Katz was partially supported by Israel Science Foundation grant no. 1517/12. S. Sanders was supported by the following funding bodies: FWO Flanders, the John Templeton Foundation, the Alexander von Humboldt Foundation, and the Japan Society for the Promotion of Science, and expresses gratitude towards these institutions. 


\section{REFERENCES}

[1] S. Albeverio, R. Høegh-Krohn, J. Fenstad and T. Lindstrøm, Nonstandard Methods in Stochastic Analysis and Mathematical Physics, Pure and Applied Mathematics, 122, Academic Press, Orlando, FL, 1986.

[2] R. Anderson, A non-standard representation for Brownian motion and Itô integration, Israel J. Math., 25 (1976), no. 1-2, 15-46.

[3] P. Andreev and E. Gordon, An axiomatics for nonstandard set theory, based on von Neumann-Bernays-Gödel theory, Journal of Symbolic Logic, 66 (2001), 1321-1341.

[4] L. Arkeryd, Intermolecular forces of infinite range and the Boltzmann equation, Archive for Rational Mechanics and Analysis, 77 (1981), no. 1, 11-21.

[5] M. Atiyah, The Interface between Mathematics and Physics: A Panel Discussion sponsored by the DIT $\&$ the RIA, Irish Mathematical Society Bulletin, 58 (2006), 33-54.

[6] J. Avigad, Weak theories of nonstandard arithmetic and analysis, Reverse mathematics 2001, Lect. Notes Log., 21, Assoc. Symbol. Logic, La Jolla, CA, $2005,19-46$.

[7] J. Bair, P. Błaszczyk, R. Ely, V. Henry, V. Kanovei, K. Katz, M. Katz, S. Kutateladze, T. McGaffey, P. Reeder, D. Schaps, D. Sherry and S. Shnider, Interpreting the infinitesimal mathematics of Leibniz and Euler, Journal for General Philosophy of Science, 48 (2017), no. 1.

See http://dx.doi.org/10.1007/s10838-016-9334-z and http://arxiv.org/abs/1605.00455

[8] J. Bair, P. Błaszczyk, R. Ely, V. Henry, V. Kanovei, K. Katz, M. Katz, S. Kutateladze, T. McGaffey, D. Schaps, D. Sherry, and S. Shnider, Is mathematical history written by the victors? Notices of the American Mathematical Society, 60 (2013) no. 7, 886-904.

See http://www .ams.org/notices/201307/rnoti-p886.pdf and http://arxiv.org/abs/1306.5973

[9] J. Bair and V. Henry, Analyse infinitésimale, Le calculus redécouvert. Academia Bruylant, Louvain-la-Neuve, 2008.

[10] D. Ballard, Foundational Aspects of 'Non'standard Mathematics, Contemporary Mathematics, 176 (1994), American Mathematical Society, Providence, R.I.

[11] D. Ballard and K. Hrbacek, Standard foundations for nonstandard analysis, Journal of Symbolic Logic, 57 (1992), 741-748.

[12] H. Barreau, "Lazare Carnot et la conception leibnizienne de l'infini mathématique." In La mathématique non standard, 43-82, Fondem. Sci., CNRS, Paris.

[13] H. Barreau, Georges Reeb et l'UPR 'Fondements des Sciences.' A propos d'un procès visant à la fois l'Analyse Non Standard et l'intuitionnisme, l'Ouvert, no. spécial G. Reeb, 1994.

[14] H. Barreau and J. Harthong (Eds.), La mathématique non standard. Histoire. Philosophie. Dossier scientifique, Fondements des Sciences, Editions du Centre National de la Recherche Scientifique (CNRS), Paris, 1989.

[15] T. Bascelli, E. Bottazzi, F. Herzberg, V. Kanovei, K. Katz, M. Katz, T. Nowik, D. Sherry and S. Shnider, Fermat, Leibniz, Euler, and the gang: The 
true history of the concepts of limit and shadow, Notices of the American Mathematical Society, 61 (2014), no. 8, 848-864.

See http://www . ams .org/notices/201408/rnoti-p848.pdf

and http://arxiv.org/abs/1407.0233

[16] T. Bascelli, P. Błaszczyk, V. Kanovei, K. Katz, M. Katz, D. Schaps and D. Sherry, Leibniz vs Ishiguro: Closing a quarter-century of syncategoremania, HOPOS: Journal of the Internatonal Society for the History of Philosophy of Science, 6 (2016), no. 1, 117-147.

See http://dx.doi.org/10.1086/685645

and http://arxiv.org/abs/1603.07209

[17] T. Bascelli, P. Błaszczyk, V. Kanovei, K. Katz, M. Katz, S. Kutateladze, T. Nowik, D. Schaps and D. Sherry, Gregory's sixth operation, Foundations of Science (2017).

See http://dx.doi.org/10.1007/s10699-016-9512-9 and https://arxiv.org/abs/1612.05944

[18] V. Benci and M. Di Nasso, Alpha-theory: an elementary axiomatics for nonstandard analysis, Expositiones Math., 21 (2003), 355-386.

[19] V. Benci, M. Forti and M. Di Nasso, The eightfold path to nonstandard analysis, Nonstandard methods and applications in mathematics, 3-44, Lect. Notes Log., 25, Assoc. Symbol. Logic, La Jolla, CA, 2006.

[20] E. Benoît, Random walks and stochastic differential equations, Nonstandard analysis in practice, 71-90, Universitext, Springer, Berlin, 1995.

[21] P. Bernays, Sur le platonisme dans les mathématiques, Enseignement Math., 34 (1935), 52-69.

[22] E. Bishop, Book Review: Elementary calculus, Bull. Amer. Math. Soc., 83 (1977), 205-208 [review of the first edition of Keisler [107]]

[23] P. Błaszczyk, A. Borovik, V. Kanovei, M. Katz, T. Kudryk, S. Kutateladze and D. Sherry, A non-standard analysis of a cultural icon: The case of Paul Halmos, Logica Universalis, 10 (2016), no. 4, 393-405.

See http://dx.doi.org/10.1007/s11787-016-0153-0 and http://arxiv.org/abs/1607.00149

[24] P. Błaszczyk, V. Kanovei, K. Katz, M. Katz, T. Kudryk, T. Mormann and D. Sherry, Is Leibnizian calculus embeddable in first order logic?, Foundations of Science, (2017), online first.

See http://dx.doi.org/10.1007/s10699-016-9495-6 and http://arxiv.org/abs/1605.03501

[25] P. Błaszczyk, V. Kanovei, K. Katz, M. Katz, S. Kutateladze and D. Sherry, Toward a history of mathematics focused on procedures, Foundations of Science, (2017).

See http://dx.doi.org/10.1007/s10699-016-9498-3

and http://arxiv.org/abs/1609.04531

[26] P. Błaszczyk, V. Kanovei, M. Katz and D. Sherry, Controversies in the foundations of analysis: Comments on Schubring's Conflicts, Foundations of Science, 22 (2017), no. 1, 125-140.

See http://dx.doi.org/10.1007/s10699-015-9473-4 and http://arxiv.org/abs/1601.00059

[27] P. Błaszczyk, M. Katz and D. Sherry, Ten misconceptions from the history of analysis and their debunking, Foundations of Science, 18 (2013), no. 1, 43-74. 
See http://dx.doi.org/10.1007/s10699-012-9285-8 and http://arxiv.org/abs/1202.4153

[28] A. Borovik and M. Katz, Who gave you the Cauchy-Weierstrass tale? The dual history of rigorous calculus, Foundations of Science, 17 (2012), no. 3, 245-276. See http://dx.doi.org/10.1007/s10699-011-9235-x

[29] J.-L. Callot, Champs lents-rapides complexes à une dimension lente, Ann. Sci. Ecole Norm. Sup. (4), 26 (1993), no. 2, 149-173.

[30] C. C. Chang and H. J. Keisler, Model theory, 3rd edition, North-Holland, 1990.

[31] A. Connes, La réalité mathématique archaïque, La Recherche, 2000. Propos recueillis par Olivier Postel-Vinay dans mensuel 332 daté juin 2000. http: //www . larecherche.fr/alain-connes-la-r\%C3\%A9alit \%C3\%A9-math\%C3\%A9matique-archa $\%$ C3 $\%$

[32] A. Connes, A. Lichnerowicz and M. Schützenberger, Triangle of thoughts, Translated from the 2000 French original by Jennifer Gage, American Mathematical Society, Providence, RI, 2001.

[33] F. S. Crawford, Jr., Waves, Berkeley Physics Course, Vol. 3, McGraw-Hill, New York, 1966.

[34] T. Cubitt, D. Perez-Garcia, M. Wolf, Undecidability of the Spectral Gap (full version), see https://arxiv.org/abs/1502.04573

[35] P. Delfini and C. Lobry, The vibrating string, Nonstandard analysis in practice, 51-70, Universitext, Springer, Berlin, 1995.

[36] F. Diener and M. Diener (Eds.), Nonstandard analysis in practice, Universitext, Springer-Verlag, Berlin, 1995.

[37] M. Diener and C. Lobry, Analyse Non Standard et représentation du réel, C.N.R.S.-Paris et O.P.U.-Alger, 1985.

[38] F. Diener and G. Reeb, Analyse non standard, Collection Enseignement des Sciences, 40. Hermann, Paris, 1989.

[39] M. Di Nasso, Iterated hyper-extensions and an idempotent ultrafilter proof of Rado's Theorem, Proceedings of the American Mathematical Society, 143 (2015), no. 4, 1749-1761.

[40] J. Earman, Infinities, infinitesimals, and indivisibles: the Leibnizian labyrinth, Studia Leibnitiana, 7 (1975), no. 2, 236-251.

[41] K. Easwaran, Regularity and hyperreal credences, Philosophical Review, 123 (2014), no. 1, 1-41.

[42] H. Edwards, Euler's definition of the derivative, Bull. Amer. Math. Soc. (N.S.), 44 (2007), no. 4, 575-580.

[43] P. Ehrlich, The rise of non-Archimedean mathematics and the roots of a misconception. I. The emergence of non-Archimedean systems of magnitudes, Archive for History of Exact Sciences, 60 (2006), no. 1, 1-121.

[44] W. Ewald (ed.), From Kant to Hilbert: a source book in the foundations of mathematics, Vol. II, Compiled, edited and with introductions by William Ewald, Oxford Science Publications, The Clarendon Press, Oxford University Press, New York, 1996.

[45] G. Ferraro, Differentials and differential coefficients in the Eulerian foundations of the calculus, Historia Mathematica, 31 (2004), no. 1, 34-61.

[46] P. Fletcher, Nonstandard set theory, Journal of Symbolic Logic, 54 (1989), 1000-1008. 
[47] M. Fliess, Analyse non standard du bruit, C. R. Math. Acad. Sci. Paris, 342 (2006), no. 10, 797-802.

[48] H. Gaifman, Nonstandard models in a broader perspective, in Ali Enayat, Roman Kossak (eds.), Nonstandard Models in Arithmetic and Set Theory, AMS Special Session Nonstandard Models of Arithmetic and Set Theory, January 15-16, 2003, Baltimore, Maryland, Contemporary Mathematics, 361, American Mathematical Society, Providence, Rhode Island, 1-22, 2004.

[49] Y. Gauthier, Towards an arithmetical logic. The arithmetical foundations of logic, Studies in Universal Logic. Birkhäuser/Springer, Cham, 2015.

[50] V. Gitman and J. Hamkins, A natural model of the multiverse axioms, Notre Dame Journal of Formal Logic, 51 (2010), no. 4, 475-484.

[51] R. Goldblatt, Lectures on the hyperreals. An introduction to nonstandard analysis, Graduate Texts in Mathematics, 188, Springer-Verlag, New York, 1998.

[52] I. Goldbring, Hilbert's fifth problem for local groups, Annals of Mathematics (2), 172 (2010), no. 2, 1269-1314.

[53] E. Gordon, Relatively nonstandard elements in the theory of internal sets of E. Nelson, Siberian Mathematical Journal, 30 (1989), 89-95.

[54] E. Gordon, Nonstandard methods in commutative harmonic analysis, American Mathematical Society, Providence, Rhode Island, 1997.

[55] E. Gordon, A. Kusraev and S. Kutateladze, Infinitesimal analysis, Updated and revised translation of the 2001 Russian original, Translated by Kutateladze, Mathematics and its Applications, 544, Kluwer Academic Publishers, Dordrecht, 2002.

[56] J. Grabiner, The origins of Cauchy's rigorous calculus, MIT Press, Cambridge, Mass.-London.

[57] J. Gray, The real and the complex: a history of analysis in the 19th century, Springer Undergraduate Mathematics Series, Springer, Cham, 2015.

[58] A. Gutman, M. Katz, T. Kudryk and S. Kutateladze, The Mathematical Intelligencer Flunks the Olympics, Foundations of Science, (2017).

See http://dx.doi.org/10.1007/s10699-016-9485-8 and http://arxiv.org/abs/1606.00160

[59] I. Hacking, Why is there philosophy of mathematics at all?, Cambridge University Press, Cambridge, 2014.

[60] P. Halmos, I want to be a mathematician. An automathography, SpringerVerlag, New York, 1985.

[61] J. Hamkins, Some second order set theory, Logic and its applications, 36-50, Lecture Notes in Comput. Sci., 5378, Springer, Berlin, 2009.

[62] J. Hamkins, The set-theoretic multiverse: a natural context for set theory, Ann. Japan Assoc. Philos. Sci., 19 (2011), 37-55.

[63] J. Hamkins, The set-theoretic multiverse, Rev. Symb. Log., 5 (2012), no. 3, 416-449.

[64] J. Hamkins, Is the dream solution of the continuum hypothesis attainable?, Notre Dame Journal of Formal Logic, 56 (2015), no. 1, 135-145.

[65] J. Hamkins, Math Overflow answer posted 17 december 2015. See http://mathoverflow.net/questions/226169

[66] J. Harthong, Études sur la mécanique quantique, Astérique 111, Société Mathématique de France, 1984. 
[67] J. Harthong and G. Reeb, Intuitionnisme 84, With comments by Harthong, Fondem. Sci., La mathématique non standard, 213-273, CNRS, Paris, 1989.

[68] J. Henle and E. Kleinberg, Infinitesimal calculus, MIT Press, Cambridge, Mass.-London, 1979.

[69] C. W. Henson, Foundations of nonstandard analysis: a gentle introduction to nonstandard extensions, Nonstandard analysis (Edinburgh, 1996), 1-49, NATO Adv. Sci. Inst. Ser. C Math. Phys. Sci., 493, Kluwer Acad. Publ., Dordrecht, 1997.

[70] C. W. Henson and H. J. Keisler, On the strength of nonstandard analysis, J. Symbolic Logic, 51 (1986), no. 2, 377-386.

[71] E. Hewitt, Rings of real-valued continuous functions. I, Transactions of the American Mathematical Society, 64 (1948), 45-99.

[72] D. Hilbert, Über das Unendliche, Math. Ann., 95 (1926), no. 1, 161-190.

[73] L. Horsten, On our ability to fix intended structures, In Moving ahead: Philosophy of Mind and Realism. Studies in the General Philosophy of Science, T. Derksen (ed.), Dutch University Press 7 (2001), 121-134.

[74] K. Hrbacek, Axiomatic foundations for nonstandard analysis, Fundamenta Mathematicae, 98 (1978), no. 1, 1-19.

[75] K. Hrbacek, Nonstandard set theory, American Mathematical Monthly, 86 (1979), no. 8, 659-677.

[76] K. Hrbacek, Internally iterated ultrapowers, In Nonstandard Models of Arithmetic and Set Theory, ed. by A. Enayat and R. Kossak, Contemporary Math. 361, American Mathematical Society, Providence, R.I., 87-120, 2004.

[77] K. Hrbacek, Nonstandard objects in set theory, in Nonstandard Methods and Applications in Mathematics, ed. by N. J. Cutland, M. Di Nasso and D. A. Ross, Lecture Notes in Logic, 25, Association for Symbolic Logic, 80-120, 2006.

[78] K. Hrbacek, Stratified analysis?, in The strength of nonstandard analysis, 4763, SpringerWienNewYork, Vienna, 2007.

[79] K. Hrbacek, Relative set theory: Internal view, Journal of Logic and Analysis, $\mathbf{1 : 8}$ (2009), 1-108.

[80] K. Hrbacek, Axiom of Choice in nonstandard set theory, Journal of Logic and Analysis, 4:9 (2012), 1-9.

[81] K. Hrbacek, O. Lessmann and R. O'Donovan, Analysis with ultrasmall numbers, Textbooks in Mathematics, CRC Press, Boca Raton, FL, 2015.

[82] H. Ishiguro, Leibniz's philosophy of logic and language. Second edition, Cambridge University Press, Cambridge, 1990.

[83] R. Jin, The sumset phenomenon, Proceedings of the American Mathematical Society, 130 (2002), no. 3, 855-861.

[84] V. Kanovei, Undecidable hypotheses in Edward Nelson's Internal Set Theory, Russian Mathematical Surveys, 46 (1991), 1-54.

[85] V. Kanovei, K. Katz, M. Katz and T. Nowik, Small oscillations of the pendulum, Euler's method, and adequality, Quantum Studies: Mathematics and Foundations, 3 (2016), no. 3, 231-236. See http://dx.doi.org/10.1007/s40509-016-0074-x and http://arxiv.org/abs/1604.06663

[86] V. Kanovei, K. Katz, M. Katz and M. Schaps, Proofs and retributions, Or: Why Sarah can't take limits, Foundations of Science, 20 (2015), no. 1, 1-25. 
See http://dx.doi.org/10.1007/s10699-013-9340-0

[87] V. Kanovei, K. Katz, M. Katz and D. Sherry, Euler's lute and Edwards' oud, The Mathematical Intelligencer, 37 (2015), no. 4, 48-51. See http://dx.doi.org/10.1007/s00283-015-9565-6 and http://arxiv.org/abs/1506.02586

[88] V. Kanovei, M. Katz and T. Mormann, Tools, Objects, and Chimeras: Connes on the Role of Hyperreals in Mathematics, Foundations of Science, 18 (2013), no. 2, 259-296. See http://dx.doi.org/10.1007/s10699-012-9316-5 and http://arxiv.org/abs/1211.0244

[89] V. Kanovei and V. Lyubetskii, Problems of set-theoretic nonstandard analysis, Uspekhi Mat. Nauk, 62 (2007), no. 1 (373), 51-122; translation in Russian Math. Surveys, 62 (2007), no. 1, 45-111.

[90] V. Kanovei and M. Reeken, Internal approach to external sets and universes. 2. External universes over the BST universe, Studia Logica, 55 (1995), no. 3, 347-376.

[91] V. Kanovei and M. Reeken, A nonstandard proof of the Jordan curve theorem, Real Analysis Exchange, 24 (1998/99), no. 1, 161-169.

[92] V. Kanovei and M. Reeken, Nonstandard analysis, axiomatically, Springer Monographs in Mathematics, Berlin, Springer, 2004.

[93] V. Kanovei and S. Shelah, A definable nonstandard model of the reals, Journal of Symbolic Logic, 69 (2004), no. 1, 159-164.

[94] K. Katz and M. Katz, Cauchy's continuum, Perspectives on Science, 19 (2011), no. 4, 426-452. See http://dx.doi.org/10.1162/POSC_a_00047 and http://arxiv.org/abs/1108.4201

[95] K. Katz and M. Katz, Meaning in classical mathematics: is it at odds with Intuitionism?, Intellectica, 56 (2011), no. 2, 223-302. See http://arxiv.org/abs/1110.5456

[96] K. Katz and M. Katz, Stevin numbers and reality, Foundations of Science, 17 (2012), no. 2, 109-123.

See http://dx.doi.org/10.1007/s10699-011-9228-9 and http://arxiv.org/abs/1107.3688

[97] M. Katz and S. Kutateladze, Edward Nelson (1932-2014), The Review of Symbolic Logic, 8 (2015), no. 3, 607-610.

See http://dx.doi.org/10.1017/S1755020315000015 and http://arxiv.org/abs/1506.01570

[98] M. Katz and E. Leichtnam, Commuting and noncommuting infinitesimals, American Mathematical Monthly, 120 (2013), no. 7, 631-641. See http://dx.doi.org/10.4169/amer.math.monthly.120.07.631 and http://arxiv.org/abs/1304.0583

[99] M. Katz and L. Polev, From Pythagoreans and Weierstrassians to true infinitesimal calculus, Journal of Humanistic Mathematics, 7 (2017), no. 1, 87-104. See http://dx.doi.org/10.5642/jhummath.201701.07 and https://arxiv.org/abs/1701.05187

[100] M. Katz, D. Schaps and S. Shnider, Almost Equal: The Method of Adequality from Diophantus to Fermat and Beyond, Perspectives on Science, 21 (2013), no. 3, 283-324. See http://dx.doi.org/10.1162/P0SC_a_00101 and http://arxiv.org/abs/1210.7750 
[101] M. Katz and D. Sherry, Leibniz's laws of continuity and homogeneity, Notices of the American Mathematical Society, 59 (2012), no. 11, 1550-1558.

See http://www . ams .org/notices/201211/rtx121101550p.pdf and http://arxiv.org/abs/1211.7188

[102] M. Katz and D. Sherry, Leibniz's infinitesimals: Their fictionality, their modern implementations, and their foes from Berkeley to Russell and beyond, Erkenntnis, 78 (2013), no. 3, 571-625.

See http://dx.doi.org/10.1007/s10670-012-9370-y and http://arxiv.org/abs/1205.0174

[103] T. Kawaï, Axiom systems of nonstandard set theory, in G. Müller at al. (Eds.), Logic Symposia, Hakone 1979, 1980, Lecture Notes in Math., 891, Springer, Berlin, 1981, 57-65.

[104] R. Kaye, Models of Peano arithmetic, Oxford Logic Guides, 15, Oxford Science Publications, The Clarendon Press, Oxford University Press, New York, 1991.

[105] H. J. Keisler, Foundations of infinitesimal Calculus. Instructor's manual, Prindle, Weber \& Schmidt, 1976.

See http://www.math.wisc.edu/ keisler/foundations.html

[106] H. J. Keisler, An infinitesimal approach to stochastic analysis, Mem. Amer. Math. Soc., 48, no. 297, 1984.

[107] H. J. Keisler, Elementary Calculus: An Infinitesimal Approach, Second Edition, Prindle, Weber and Schmidt, Boston, 1986. See http://www.math.wisc.edu/ keisler/calc.html

[108] S. Kleene, Mathematical logic, John Wiley \& Sons, New York-LondonSydney, 1967.

[109] U. Kohlenbach, Applied proof theory: proof interpretations and their use in mathematics, Springer Monographs in Mathematics, Springer-Verlag, Berlin, 2008.

[110] L. Kronecker, Über den Zahlbegriff, Journal für die reine und angewandte Mathematik, 101 (1887), 337-355. Reprinted in Kronecker, L. (1899), Werke, 5 vols, K. Hensel (ed), Teubner, Leipzig, vol. 3, 251-274.

[111] D. Laugwitz, Infinitely small quantities in Cauchy's textbooks, Historia Mathematica, 14 (1987), 258-274.

[112] A. Lightstone and A. Robinson, Nonarchimedean fields and asymptotic expansions, North-Holland Mathematical Library, 13, North-Holland, AmsterdamOxford; American Elsevier Publishing, New York, 1975.

[113] T. Lindstrøm, An invitation to nonstandard analysis, in Nonstandard analysis and its applications (Hull, 1986), 1-105, London Math. Soc. Stud. Texts, 10, Cambridge Univ. Press, Cambridge, 1988.

[114] C. Lobry, Et pourtant... ils ne remplissent pas $\mathbb{N}$, With a preface by Renée Peiffer-Reuter, Aléas, Lyon, 1989.

[115] C. Lobry, Traces macroscopiques de marches de pas infiniment petits, in J.M. Salanskis and H. Sinaceur, editors, Le labyrinthe du continu, 436-444, Springer Verlag, 1992.

[116] C. Lobry and T. Sari, Equations différentielles à second membres discontinus, Ecole du CIMPA, Quelques Questions de Théorie du Contrôle, Hermann, 2004. 
[117] P. Loeb, Conversion from nonstandard to standard measure spaces and applications in probability theory, Trans. Amer. Math. Soc., 211 (1075), 113-122.

[118] P. Loeb and M. Wolff (Eds.), Nonstandard Analysis for the Working Mathematician, Second Edition, Springer, Dordrecht, 2015.

[119] J. Łoś, Quelques remarques, théorèmes et problèmes sur les classes défnissables d'algèbres, in Mathematical interpretation of formal systems, 98-113, North-Holland Publishing, Amsterdam, 1955.

[120] R. Lutz and M. Goze, Nonstandard analysis. A practical guide with applications. With a foreword by Georges H. Reeb, Lecture Notes in Mathematics, 881, Springer-Verlag, Berlin-New York, 1981.

[121] W. Luxemburg, Non-standard analysis, lecture notes, Department of Mathematics, California Institute of Technology, 1962.

[122] P. Maddy, Realism in mathematics, The Clarendon Press, Oxford University Press, New York, 1990.

[123] P. Mancosu, Measuring the size of infinite collections of natural numbers: was Cantor's theory of infinite number inevitable?, Rev. Symb. Log., 2 (2009), no. $4,612-646$.

[124] M. Mandelkern, Brouwerian counterexamples, Math. Mag., 62 (1989), no. 1, $3-27$.

[125] P. Martin-Löf, Mathematics of infinity, COLOG-88 (Tallinn, 1988), 146-197, Lecture Notes in Comput. Sci., 417, Springer, Berlin, 1990.

[126] V. Molchanov, The use of double nonstandard enlargements to topology, Siberian Mathematical Journal, 30 (1989), no. 3, 397-402.

[127] E. Nelson, Internal set theory: a new approach to nonstandard analysis, Bulletin of the American Mathematical Society, 83 (1977), no. 6, 1165-1198.

[128] E. Nelson, Radically elementary probability theory, Annals of Mathematics Studies, 117. Princeton University Press, Princeton, NJ, 1987.

[129] T. Nowik and M. Katz, Differential geometry via infinitesimal displacements, Journal of Logic and Analysis, 7:5 (2015), 1-44.

See http://dx.doi.org/10.4115/jla.2015.7.5 and http://arxiv.org/abs/1405.0984

[130] H. Osswald and S. Sanders, To be or not to be constructive, in 'L.E.J. Brouwer, fifty years later', Brouwer symposium 2016, special issue of Indagationes Mathematicae, 2017.

[131] E. Palmgren, A bibliography of constructive nonstandard mathematics, 1996, see http://www2.math.uu.se/\%7Epalmgren/biblio/nonstd.html

[132] Y. Péraire, Theorie relative des ensembles internes, Osaka Journal of Mathematics, 29 (1992), 267-297.

[133] Y. Péraire, Infinitesimal approach of almost-automorphic functions, Annals of Pure and Applied Logic, 63 (1993), 283-297.

[134] Y. Péraire and G. Wallet, Une theorie relative des ensembles internes, Comptes Rendus de l'Academie des Sciences, Paris, Serie I, Mathematique, 308 (1989), 301-304.

[135] D. Pražák and J. Slavík, Nonstandard analysis of global attractors, MLQ. Mathematical Logic Quarterly, 61 (2015), no. 4-5, 315-328.

[136] H. Putnam, Models and reality, J. Symbolic Logic, 45 (1980), no. 3, 464-482.

[137] F. Quinn, A revolution in mathematics? What really happened a century ago and why it matters today. Notices Amer. Math. Soc. 59 (2012), no. 1, 31-37. 
[138] P. Quinon and K. Zdanowski, The Intended Model of Arithmetic. An Argument from Tennenbaum's Theorem, in S Barry Cooper, Thomas F. Kent, Benedikt Löwe, Andrea Sorbi (Eds.), Computation and Logic in the Real World. Third Conference on Computability in Europe, CiE 2007, Siena, Italy, June 18-23, 2007, 313-317.

[139] G. Reeb, La mathématique non standard vieille de soixante ans?, Publications de l'Institut de Recherche Mathématique avancée 64/P-30, Université de Strasbourg, 1979 (reprinted in Salanskis [148])

[140] G. Reeb, La mathématique non standard vieille de soixante ans?, Third Colloquium on Categories, Part III (Amiens, 1980), Cahiers Topologie Géom. Différentielle, 22 (1981), no. 2, 149-154.

[141] A. Robinson, Non-standard analysis, North-Holland Publishing, Amsterdam, 1966.

[142] A. Robinson, Concerning progress in the philosophy of mathematics, Logic Colloquium 1973 (Bristol, 1973), pp. 41-52, Studies in Logic and the Foundations of Mathematics, 80, North-Holland, Amsterdam, 1975, reprinted in Selected Papers [143, p. 557].

[143] A. Robinson, Selected papers of Abraham Robinson, Vol. II, Nonstandard analysis and philosophy, Edited and with introductions by W. A. J. Luxemburg and S. Körner, Yale University Press, New Haven, Conn., 1979.

[144] A. Robinson and E. Zakon, A set-theoretical characterization of enlargements, in Applications of model theory to algebra, analysis and probability, W. A. J. Luxemburg, ed., Holt, Rinehart and Winston, 109-122, 1969.

[145] D. Ross, Loeb measure and probability, in Nonstandard analysis (Edinburgh, 1996), 91-120, NATO Adv. Sci. Inst. Ser. C Math. Phys. Sci., 493, Kluwer Acad. Publ., Dordrecht, 1997.

[146] J. E. Rubio, Optimization and nonstandard analysis, Monographs and Textbooks in Pure and Applied Mathematics 184, Marcel Dekker, New York, 1994.

[147] J.-M. Salanskis, Un maître, L'Ouvert, no. spécial G. Reeb, 1994.

[148] J.-M. Salanskis, Le constructivisme non standard, Histoire des Sciences, Presses Universitaires du Septentrion, Villeneuve d'Ascq, 1999.

[149] S. Sanders, The unreasonable effectiveness of Nonstandard Analysis. See http://arxiv.org/abs/1508.07434

[150] S. Sanders, Reverse Formalism 16, Synthese, 2017, Special Issue for SOTFOMIII, to appear. See http://dx.doi.org/10.1007/s11229-017-1322-2 and https://arxiv.org/abs/1701.05066

[151] G. Schubring, Conflicts between generalization, rigor, and intuition. Number concepts underlying the development of analysis in 17-19th Century France and Germany, Sources and Studies in the History of Mathematics and Physical Sciences, Springer-Verlag, New York, 2005.

[152] Y. Sergeyev, The Olympic Medals Ranks, Lexicographic Ordering, and Numerical Infinities, The Mathematical Intelligencer, 37 (2015), no. 2, 4-8.

[153] D. Sherry and M. Katz, Infinitesimals, imaginaries, ideals, and fictions, Studia Leibnitiana, 44 (2012), no. 2, 166-192.

See http://www.jstor.org/stable/43695539

and http://arxiv.org/abs/1304.2137 Article was published in 2014 (even though the journal issue lists the year as 2012) 
[154] T. Skolem, Über die Unmöglichkeit einer vollständigen Charakterisierung der Zahlenreihe mittels eines endlichen Axiomensystems, Norsk Mat. Forenings Skr., II. Ser., no. 1/12 (1933), 73-82.

[155] A. Sochor, The alternative set theory, in Set Theory and Hierarchy Theory, Lecture Notes in Mathematics, 537, Springer, Berlin, 259-273, 1976.

[156] D. Spalt, Cauchys Kontinuum. Eine historiografische Annäherung via Cauchys Summensatz [Cauchy's continuum. A historiographic approach via the Cauchy sum theorem], Arch. Hist. Exact Sci., 56 (2002), no. 4, 285-338.

[157] K. Stroyan and W. Luxemburg, Introduction to the theory of infinitesimals, Pure and Applied Mathematics, 72, Academic Press [Harcourt Brace Jovanovich, Publishers], New York-London, 1976.

[158] T. Tao, Structure and randomness. Pages from year one of a mathematical blog, American Mathematical Society, Providence, RI, 2008.

[159] T. Tao, Compactness and contradiction, American Mathematical Society, Providence, RI, 2013.

[160] T. Tao, Hilbert's fifth problem and related topics, Graduate Studies in Mathematics, 153, American Mathematical Society, Providence, RI, 2014.

[161] T. Tao and V. Vu, Sum-avoiding sets in groups, Discrete Analysis, 2016:15, $31 \mathrm{pp}$, see http://dx.doi.org/10.19086/da.887 and http://arxiv.org/abs/1603.03068

[162] B. van den Berg, E. Briseid and P. Safarik, A functional interpretation for nonstandard arithmetic, Ann. Pure Appl. Logic, 163 (2012), no. 12, 19621994.

[163] I. van den Berg, Nonstandard asymptotic analysis, Lecture Notes in Mathematics, 1249, Springer-Verlag, Berlin, 1987.

[164] I. van den Berg, Principles of infinitesimal stochastic and financial analysis, World Scientific Publishing, River Edge, NJ, 2000.

[165] L. van den Dries and I. Goldbring, Hilbert's 5th problem, Enseign. Math., 61 (2015), no. 1-2, 3-43.

[166] P. Vopěnka, Mathematics in the Alternative Set Theory, Teubner Texte, Leipzig, 1979.

[167] P. Vopěnka and P. Hájek, The theory of semisets, Academia (Publishing House of the Czechoslovak Academy of Sciences), Prague, 1972.

[168] G. Wallet, Entrée-sortie dans un tourbillon, Ann. Inst. Fourier (Grenoble), 36 (1986), no. 4, 157-184.

[169] H. Wang, Metalogic, Encyclopedia Britannica online, 2016, see http://www . britannica.com/topic/metalogic

[170] H. Weisshaupt, Diffusion processes via parabolic equations: an infinitesimal approach to Lindeberg's limit theorem, Journal of Logic and Analysis, 1 (2009), Paper 2, 29 pp.

[171] H. Weisshaupt, Radically elementary analysis of an interacting particle system at an unstable equilibrium, Journal of Logic and Analysis, 3 (2011), Paper 10, $47 \mathrm{pp}$.

[172] R. Werner and P. Wolff, Classical mechanics as quantum mechanics with infinitesimal $\hbar$, Physics Letters A, 202 (1995), 155-159. 
P. Fletcher, School of Computing and Mathematics, Keele UniverSity, Keele, Staffordshire, ST5 2BG, UK

E-mail address: p.fletcher@keele.ac.uk

K. Hrbacek, 305 West 98 Street, Apt 4AS, New York, NY 10025, U.S.A.

E-mail address: khrbacek@icloud.com

V. Kanovei, Laboratory 6, IPPI Ran, Bolshoy Karetny Per. 19, Build. 1, Moscow 127051, Russia; and MIIT, 9в9 Obrazcova St, 127994 Moscow, Russia

E-mail address: kanovei@googlemail.com

M. Katz, Department of Mathematics, Bar Ilan University, Ramat GAN 52900 ISRAEL

E-mail address: katzmik@macs.biu.ac.il

C. Lobry, Centre de Recherche en Histoire des Idées (CRHi), Université de Nice Sophia Antipolis, 98, Bd Édouard Herriot, BP 3209 06204 NiCE CEDEX

E-mail address: claude.lobry@icloud.com

S. Sanders, Ludwig-Maximilians-Universität MÜnchen, Fakultät Für Philosophie, Wissenschaftstheorie und Religionswissenschaft, Munich Center for Mathematical Philosophy, Geschwister-Scholl-Platz 1, D80539 München, Germany

E-mail address: sasander@me.com 